\title{
An incremental approach for attribute reduction based on knowledge granularity
}

\author{
Yunge Jing ${ }^{\mathrm{a}, \mathrm{b}}$, Tianrui Li $\mathrm{Li}^{\mathrm{a}, *}$, Chuan Luo ${ }^{\mathrm{c}}$, Shi-Jinn Horng ${ }^{\mathrm{d}}$, Guoyin Wang ${ }^{\mathrm{e}}$, Zeng $\mathrm{Yu}^{\mathrm{a}}$ \\ ${ }^{a}$ School of Information Science and Technology, Southwest Jiaotong University, Chengdu 610031, China \\ ${ }^{b}$ Department of Public Computer Teaching, Yuncheng University, Yuncheng 044000, China \\ ${ }^{c}$ College of Computer Science, Sichuan University, Chengdu 610065, China \\ ${ }^{d}$ Department of Computer Science and Information Engineering, National Taiwan University of Science and Technology, Taipei 106, Taiwan \\ ${ }^{e}$ Chongqing Key Laboratory of Computational Intelligence, Chongqing University of Posts and Telecommunications, Chongqing 400065, China
}

\begin{abstract}
Rough set provides a theoretical framework for classification learning in data mining and knowledge discovery. As an important application of rough set, attribute reduction, also called feature selection, aims to reduce the redundant attributes in a given decision table while preserving a particular classification property, e.g., information entropy and knowledge granularity. In view of the dynamically varying object set in an decision table, in this paper, we focus on knowledge granularity-based attribute reduction approach with incremental objects. Incremental mechanisms for updating knowledge granularity are introduced. The corresponding incremental algorithms for attribute reduction when adding multiple objects are then developed. Experiments conducted on different data sets from UCI show that the proposed incremental algorithms can achieve better performance than the non-incremental counterpart and incremental algorithms based on entropies.
\end{abstract}

Keywords: Decision table, Incremental learning, knowledge granularity, attribute reduction, rough set theory.

\section{Introduction}

Rough Set Theory (RST), as an important soft computing tool to discover the potentially useful knowledge from databases, has attracted much attention. Nowadays, it has become a common technique in many research areas such as machine learning [1], pattern recognition [2], decision supporting [3, 4], expert system [5], data mining and knowledge discovery $[6-8,25]$.

Attribute reduction is an important concept in RST, which is able to select useful attributes from a given decision system. Attribute reduction keeps the distinguishing power of original decision system. Many methods for attribute reduction have been developed in the last two decades. However, these approaches are often applied in static decision systems $[13,15,21,24,25,31,32]$. As objects increase dynamically in a given decision system, non-incremental reduction algorithms often need to compute reduction repeatedly and thus consume a great deal of computational time. Hence, these reduction algorithms are very inefficient to deal with dynamic decision systems.

To overcome this deficiency, an incremental learning based on RST is an effective method to deal with dynamic data sets $[29,30]$. These algorithms usually can carry out the computation using the existing results from the original data set. And they have been successfully applied to data analysis in real-time applications. Researchers have proposed many incremental algorithms to update approximations, reduct and decision rules in dynamic information systems which can be divided into three aspects as follows. 1) Incremental updating algorithms on the change of the attribute set. Based on upper and lower boundary sets, Chan developed an incremental algorithm for updating upper and lower approximations. It is an effective method to deal with dynamic attribute sets [9]. Li et al. proposed an incremental method for fast computing approximations by the characteristic relation when the attribute set varies in incomplete information systems [10]. Zhang et al. developed a matrix-based incremental algorithm to fast compute

\footnotetext{
${ }^{*}$ Corresponding author.

Email addresses: jyg701022@163.com (Yunge Jing), trli@swjtu.edu.cn (Tianrui Li ), cluo@scu.edu.cn (Chuan Luo), horngsj@yahoo.com.tw (Shi-Jinn Horng), wanggy@ieee.org (Guoyin Wang), yuzeng2005@163.com (Zeng Yu)
} 
approximations under the variation of the attribute set in set-valued information systems [11]. Cheng developed two incremental approaches to compute approximations based on cut sets and boundary sets, respectively [12]. Wang et al. developed a dimension incremental algorithm based on information entropy for updating the attribute reduction, and then proposed an algorithm that can find a new reduction efficiently when an attribute set was added to a decision table [13]. In [14], Li et al. introduced the dominance matrix to compute dominating and dominated sets, and proposed an incremental method for updating approximations when the set of attributes varies. Luo et al. developed an incremental approach to update relevant matrices, and presented incremental methods for computing approximations in set-valued information systems [26]. For incomplete decision systems, Shu et al. developed a positive region-based attribute reduction approach to update the attribute reduction problem efficiently with dynamically varying attribute set [28]. 2) Incremental updating algorithms on the change of the object set. Liu et al. presented an incremental attribute reduction algorithm to compute the minimal reduction in the information systems without the decision attribute [15]. Orlowska et al. presented an incremental reduction method for updating the attribute reduction in a decision table [16]. Considering that the presented method is very time-consuming, Hu et al. proposed an effective incremental reduction algorithm based on the positive region to overcome this deficiency [17]. Liang et al. developed a group incremental attribute reduction algorithm based on information entropy when adding multiple objects into a decision table [18]. Yang et al. proposed an incremental method to update attribute reduction based on the discernibility matrix in the decision systems [19]. 3) Incremental updating algorithms on the change of the attribute values. Chen et al. introduced the definitions of refining and coarsening, and developed the incremental approaches for computing approximations when coarsening or refining attribute values in single-valued information systems [20]. Based on three representative entropies, Wang et al. presented an attribute reduction algorithm for data sets with dynamically varying attribute values [21]. Chen et al. introduced the definition of minimal discernibility attribute set, which focuses on improving the efficiency of attribute reduction. Furthermore, they developed a rough set-based approach for updating decision rules in inconsistent information systems [27].

The knowledge granularity has become one of the most common uncertain measures of data sets. It has been widely applied to construct heuristic reduction approaches [23, 24, 31]. From the above discussion, most literatures discussed incremental reduction algorithms based on information entropy or positive region to maintain reduction when data varying dynamically. However, there is no report of incremental reduction algorithm with knowledge granularity to update reduction under the dynamic data environment. In order to update reduction effectively in the decision table, it is desirable and interesting to investigate incremental reduction method based on knowledge granularity in dealing with dynamic data. Matrix is a very useful and handy tool in computing. It can be computed intuitively and operated easily for information systems. Hence, we first develop a matrix-based updating attribute reduction with the variation of objects under knowledge granularity in the paper. However, in real applications, matrixbased methods need more space, which lead to consume a great deal of computational time. Then, an incremental attribute reduction based on non-matrix is proposed to deal with such data sets. Finally, the experimental results verify the proposed incremental reduction algorithm can achieve better performance than incremental algorithm based on information entropies and the non-incremental counterpart.

The remainder of this paper is organized as follows. Section 2 briefly reviews some basic concepts in RST. Section 3 introduces the representation of the knowledge granularity and a general heuristic reduction algorithm based on knowledge granularity. Incremental reduction algorithms based on matrix and non-matrix when adding multiple objects are introduced in Section 4. In Section 5, experimental evaluation on nine UCI data sets is outlined to demonstrate the effectiveness and efficiency of the proposed algorithm. The paper ends with conclusions in Section 6.

\section{Preliminaries}

We review some basic concepts of information systems and RST in this subsection[25].

Definition 1. [25]An information system is a quadruple tuple $S=(U, A, V, f)$, where $U$ is a finite non-empty object set and $A$ is a finite nonempty attribute set, $V=\cup_{a \in A} V_{a}, V_{a}$ is a domain of attribute a, and $f: U \times A \rightarrow V$ is an information function with $f(x, a) \in V_{a}$ for each $a \in A$ and $x \in U$. If $A=C \cup D$, where $C$ is the conditional attribute set and $D$ is the decision attribute set, then $S=(U, A, V, f)$ is also called as a decision table. 
Definition 2. [25]An indiscernibility (equivalence) relation is determined by each nonempty subset $B \subseteq$ A as follows:

$$
R_{B}=\{(x, y) \in U \times U \mid f(x, a)=f(y, a), \forall a \in B\}
$$

The universe $U$ is divided into some equivalence classes by the indiscernibility relation $R_{B}$, given by $U / R_{B}=$ $\left\{[x]_{B} \mid x \in U\right\}$, and the equivalence class including the object $x$ with respect to $B$ is denoted as $[x]_{B}=\left\{y \in U \mid(x, y) \in R_{B}\right\}$.

Definition 3. [25]Given an information system $S=(U, A, V, f)$ and $X \subseteq U . R$ is an equivalence relation. The lower and upper approximations of $X$ with respect to $R$ are defined as follows, respectively:

$$
\begin{gathered}
\underline{R} X=\cup\left\{x \in U \mid[x]_{R} \subseteq X\right\}, \\
\bar{R} X=\cup\left\{x \in U \mid[x]_{R} \cap X \neq \varnothing\right\} .
\end{gathered}
$$

The universe $U$ is partitioned into three disjoint regions by these two approximations $(\underline{R} X, \bar{R} X)$ : the negative region $N E G_{R}(X)$, the boundary region $B N D_{R}(X)$, and the positive region $P O S_{R}(X)$. Then the three regions are defined as follows, respectively[25]:

$$
\left\{\begin{array}{l}
N E G_{R}(X)=U-\bar{R} X \\
B N D_{R}(X)=\bar{R} X-\underline{R} X \\
\operatorname{POS}_{R}(X)=\underline{R} X
\end{array}\right.
$$

\section{ATTRIBUTE REDUCTION BASED ON KNOWLEDGE GRANULARITY}

This section reviews some basic concepts of knowledge granularity and relation matrix. Then some properties are obtained and a general heuristic attribute reduction algorithm based on knowledge granularity for decision tables is introduced.

\subsection{A representation of the knowledge granularity}

Definition 4. [23]Let $S=(U, C \cup D, V, f)$ be a decision table and $U / C=\left\{X_{1}, X_{2}, \cdots, X_{m}\right\}$. Based on the partition, a knowledge granularity of $C$ is defined as:

$$
G P_{U}(C)=\sum_{i=1}^{m} \frac{\left|X_{i}\right|^{2}}{|U|^{2}}
$$

The heuristic attribute reduction algorithms based on knowledge granularity aim to preserve the relative knowledge granularity unchanged. Then the relative knowledge granularity shown as follows was applied to construct heuristic reduction algorithms and delete the redundant attributes in a decision table [25].

Definition 5. [24]Let $S=(U, C \cup D, V, f)$ be a decision table. $U / C=\left\{X_{1}, X_{2}, \cdots, X_{m}\right\}$ and $U /(C \cup D)=$ $\left\{Y_{1}, Y_{2}, \cdots, Y_{n}\right\}$. Based on these partitions, a knowledge granularity of $C$ relative to $D$ is defined as follows:

$$
G P_{U}(D \mid C)=G P_{U}(C)-G P_{U}(C \cup D)
$$

The attribute significance measure based the knowledge granularity in a heuristic reduction is shown as follows.

Definition 6. [24]Let $S=(U, C \cup D, V, f)$ be a decision table. $U / C=\left\{X_{1}, X_{2}, \cdots, X_{m}\right\}$ and $B \subseteq C . \forall a \in B$, the significance measure (inner significance) of a in $B$ is defined as:

$$
S i g_{U}^{\text {inner }}(a, B, D)=G P_{U}(D \mid(B-\{a\}))-G P_{U}(D \mid B)
$$

Definition 7. [24]Let $S=(U, C \cup D, V, f)$ be a decision table and $B \subseteq C . \forall a \in(C-B)$, the significance measure (outer significance) of a in $B$ is defined as:

$$
S i g_{U}^{\text {outer }}(a, B, D)=G P_{U}(D \mid B)-G P_{U}(D \mid(B \cup\{a\}))
$$


Given a decision table $S=(U, A, V, f)$ and $a \in C$. If $S_{i} g_{U}^{\text {inner }}(a, B, D)>0$, one can obtain that the attribute $a$ is indispensable and a core attribute of $S$. On the basis of the core attributes, an attribute reduction can be obtained by gradually adding selected attributes with the highest significance to the core through a heuristic attribute reduction algorithm. The reduction based on knowledge granularity of $C$ relative to $D$ is shown as follows $[18,24,32]$.

Definition 8. [25]Let $S=(U, C \cup D, V, f)$ be a decision table and $B \subseteq C$. Then $B$ is a relative reduction based on the knowledge granularity of $S$ if:

(1) $G P_{U}(D \mid B)=G P_{U}(D \mid C)$ and

(2) $\forall a \in B, G P_{U}(D \mid(B-\{a\})) \neq G P_{U}(D \mid B)$.

\subsection{The matrix presentation of the knowledge granularity}

Definition 9. [11]Let $S=(U, C \cup D, V, f)$ be a decision table and $U / C=\left\{X_{1}, X_{2}, \cdots, X_{m}\right\} . R_{C}$ is an equivalence relation on $U$. Then a relation matrix $\left(M_{U}^{R_{C}}\right)_{n \times n}=\left(m_{i j}\right)_{n \times n}$ is defined as:

$$
m_{i j}=\left\{\begin{array}{cc}
1, & \left(u_{i}, u_{j}\right) \in R_{C} \\
0, & \left(u_{i}, u_{j}\right) \notin R_{C}
\end{array} \quad 1 \leq i, j \leq n\right.
$$

For simplicity, $\left(M_{U}^{R_{C}}\right)_{n \times n}$ is written as $\left(M_{U}^{R_{C}}\right)$ in the following.

Definition 10. [31] Let $S=(U, C \cup D, V, f)$ be a decision table and $\left(M_{U}^{R_{C}}\right)_{n \times n}=\left(m_{i j}\right)_{n \times n}$ be an $n \times n$ relation matrix for $R_{C}$. Based on the relation matrix, a knowledge granularity of $C$ is defined as:

$$
G P_{U}(C)=\sum_{i=1}^{n} \sum_{j=1}^{n} \frac{m_{i j}}{n^{2}}=\frac{S u m\left(M_{U}^{R_{C}}\right)}{n^{2}}=\overline{M_{U}^{R_{C}}}
$$

where $n$ stands for the cardinal number of $U . S$ um $\left(M_{U}^{R_{C}}\right)$ stands for the summation of all elements of the matrix $M_{U}^{R_{C}}$, and $\overline{M_{U}^{R_{C}}}$ stands for the average of matrix $M_{U}^{R_{C}}$.

Following is an example to explain how to compute the knowledge granularity based on the relation matrix.

Example 1. Table 1 is a decision table. $U=\{1,2,3,4,5,6,7,8,9\}$ is the universe, $C=\{a, b, c, e, f\}$ is the condition attribute set and $D=\{d\}$ is the decision attribute.

Table 1: A Decision Table.

\begin{tabular}{lllllll}
\hline $\mathrm{u}$ & $\mathrm{a}$ & $\mathrm{b}$ & $\mathrm{c}$ & $\mathrm{e}$ & $\mathrm{f}$ & $\mathrm{d}$ \\
\hline 1 & 1 & 0 & 0 & 0 & 1 & 0 \\
2 & 0 & 0 & 1 & 0 & 1 & 0 \\
3 & 0 & 1 & 1 & 1 & 0 & 1 \\
4 & 0 & 0 & 1 & 0 & 1 & 0 \\
5 & 0 & 1 & 1 & 0 & 0 & 1 \\
6 & 1 & 0 & 0 & 0 & 0 & 1 \\
7 & 1 & 0 & 1 & 0 & 0 & 1 \\
8 & 0 & 1 & 1 & 0 & 1 & 1 \\
9 & 0 & 1 & & 0 & & 1 \\
\hline
\end{tabular}


According to Definitions 9 and 10, we have $U / C=\{\{1\},\{2,4\},\{3,5\},\{6,7\},\{8,9\}\}$,

$$
\begin{gathered}
M_{U}^{R_{C}}=\left[\begin{array}{ccccccccc}
1 & 0 & 0 & 0 & 0 & 0 & 0 & 0 & 0 \\
0 & 1 & 0 & 1 & 0 & 0 & 0 & 0 & 0 \\
0 & 0 & 1 & 0 & 1 & 0 & 0 & 0 & 0 \\
0 & 1 & 0 & 1 & 0 & 0 & 0 & 0 & 0 \\
0 & 0 & 1 & 0 & 1 & 0 & 0 & 0 & 0 \\
0 & 0 & 0 & 0 & 0 & 1 & 1 & 0 & 0 \\
0 & 0 & 0 & 0 & 0 & 1 & 1 & 0 & 0 \\
0 & 0 & 0 & 0 & 0 & 0 & 0 & 1 & 1 \\
0 & 0 & 0 & 0 & 0 & 0 & 0 & 1 & 1
\end{array}\right], \\
G P_{U}(C)=\overline{M_{U}^{R_{C}}}=\frac{1}{81} \times \operatorname{Sum}\left(M_{U}^{R_{C}}\right)=\frac{17}{81} .
\end{gathered}
$$

Definition 11. Let $S=(U, C \cup D, V, f)$ be a decision table. $M_{U}^{R_{C}}$ and $M_{U}^{R_{C u D}}$ are the relation matrices for $R_{C}$ and $R_{(C \cup D)}$, respectively. Based on these relation matrices, a knowledge granularity of $C$ relative to $D$ is defined as:

$$
G P_{U}(D \mid C)=\overline{M_{U}^{R_{C}}}-\overline{M_{U}^{R_{(C \cup D)}}}
$$

Definition 12. Let $S=(U, C \cup D, V, f)$ be a decision table. $M_{U}^{R_{C}}, M_{U}^{\left(R_{C-(a))}\right.}, M_{U}^{R_{(C \cup D)}}$ and $M_{U}^{R_{((C-(a) \cup D)}}$ are the relation matrices for $R_{C}, R_{(C-\{a\})}, R_{(C \cup D)}$ and $R_{((C-\{a\}) \cup D)}$, respectively. Based on these relation matrices, the significance measure (inner significance) of a in $C$ is defined as:

$$
\operatorname{Sig}_{U}^{\text {inner }}(a, C, D)=\overline{M_{U}^{R_{(C-(a))}}}-\overline{M_{U}^{R_{((C-(a)) \cup D)}}}-\overline{M_{U}^{R_{C}}}+\overline{M_{U}^{R_{(C \cup D)}}}
$$

Definition 13. Let $S=(U, C \cup D, V, f)$ be a decision table. The core of $S$ is defined as:

$$
\text { Core }_{C}=\left\{a \in C \mid S i g_{U}^{\text {inner }}(a, C, D)>0\right\}
$$

Example 2. According to Definition 12 and 13 , we can get

$$
\begin{array}{r}
\operatorname{Sig}_{U}^{\text {inner }}(a, C, D)=\overline{M_{U}^{R_{(C-(a))}}}-\overline{M_{U}^{R_{((C-l a) \cup D)}}}-\overline{M_{U}^{R_{C}}}+\overline{M_{U}^{R_{(C \cup D)}}}=0, \operatorname{Sig}_{U}^{\text {inner }}(b, C, D)=0, \\
\operatorname{Sig}_{U}^{\text {inner }}(c, C, D)=0, \quad \operatorname{Sig}_{U}^{\text {inner }}(e, C, D)=0, \quad \operatorname{Sig}_{U}^{\text {inner }}(f, C, D)=\frac{4}{81}>0 .
\end{array}
$$

Then, we have Core $_{C}=\{f\}$.

Definition 14. Let $S=(U, C \cup D, V, f)$ be a decision table. $C_{0} \subseteq C . M_{U}^{R_{C_{0}}}, M_{U}^{R_{\left(C_{0} \cup D\right)}}, M_{U}^{R_{\left(C_{0} \cup(a)\right)}}$ and $M_{U}^{R_{\left(\left(C_{0} \cup(a) \cup D\right)\right.}}$ are the relation matrices for $R_{C_{0}}, R_{\left(C_{0} \cup D\right)}, R_{\left(C_{0} \cup\{a\}\right)}$ and $R_{\left(\left(C_{0} \cup\{a\}\right) \cup D\right)}$, respectively. Based on these relation matrices, $\forall a \in\left(C-C_{0}\right)$, the significance measure (outer significance) of $a$ in $C_{0}$ is defined as:

$$
\operatorname{Sig}_{U}^{\text {outer }}\left(a, C_{0}, D\right)=\overline{M_{U}^{R_{C_{0}}}}-\overline{M_{U}^{R_{\left(C_{0} \cup D\right)}}}-\overline{M_{U}^{R_{\left(C_{0} \cup(a)\right)}}}+\overline{M_{U}^{R_{\left(\left(C_{0} \cup(a) \cup D\right)\right.}}}
$$

Since the heuristic attribute reduction strategies of the two algorithms in matrix and non-matrix, respectively, are similar to each other, a general heuristic attribute reduction algorithm based on knowledge granularity for decision tables is introduced in Algorithm 1 [13, 18, 21, 25].

Following is the time complexity analysis of Algorithm 1. Given a decision table, according to Definition 4, we first compute conditional classes and decision classes, respectively. Then, the value of knowledge granularity is computed. Xu et al. in [22] presented a fast algorithm for the partition with a time complexity being $O(|C||U|)$. Hence, the time complexities of classic heuristic attribute reduction algorithms for computing knowledge granularity are $O(|C||U|+|m|) \approx O(|C||U|)$. Therefore, the time complexity of computing the core for the decision table Steps 3-7 is $O\left(|C||U|^{2}\right)$, the time complexity of Steps 10-15 is $O\left(|C||U|^{2}\right)$, and the time complexity of Steps 17-21 is $O\left(|C||U|^{2}\right)$. Hence, the time complexity of classic heuristic attribute reduction algorithm for computing the reduction is $O\left(|C||U|^{2}+\right.$ $\left.|C||U|^{2}+|C||U|^{2}+|C||U|+|m|\right) \approx O\left(\left.|C \|| U\right|^{2}\right)$. 


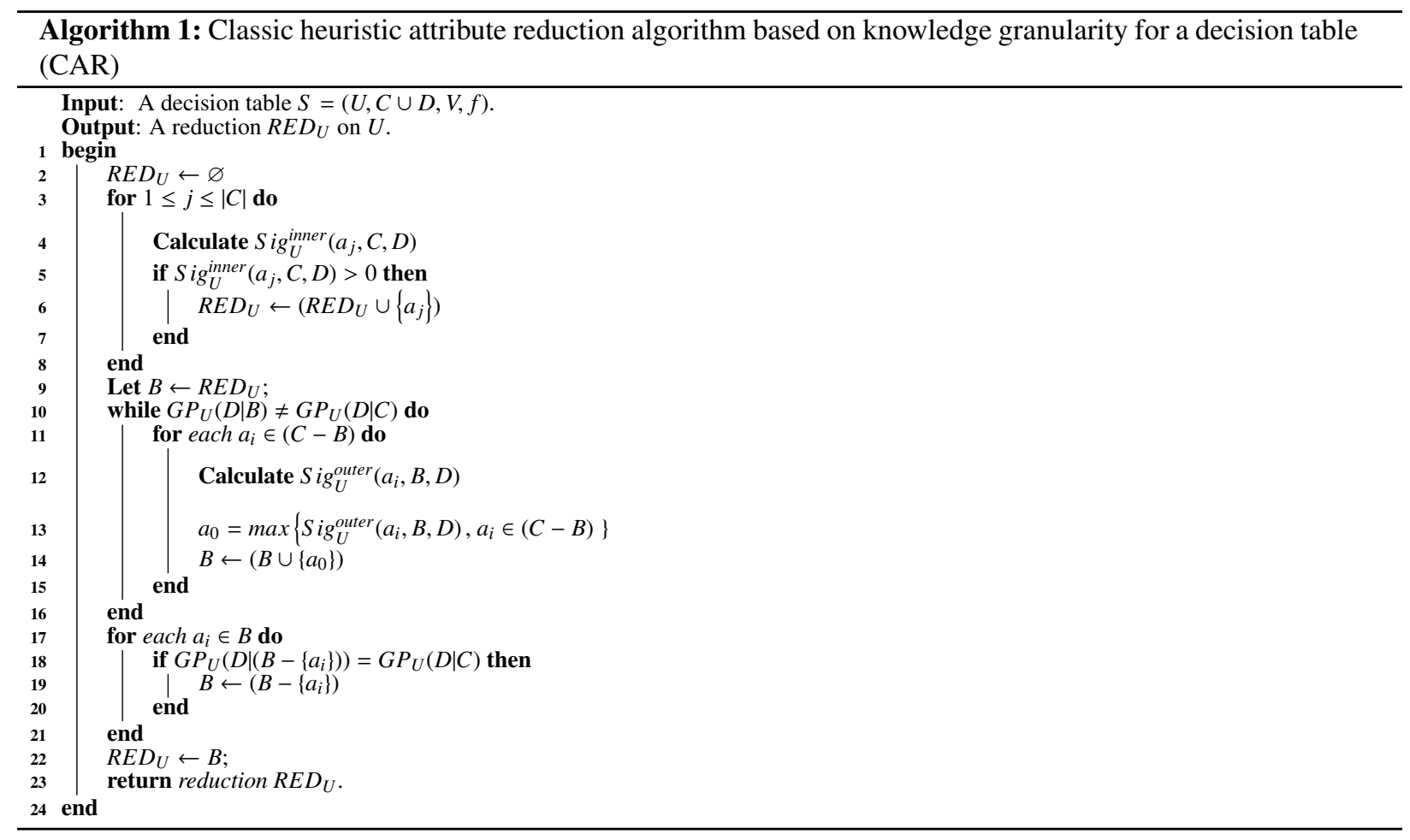

\section{INCREMENTAL ATTRIBUTE REDUCTION ALGORITHM WHEN ADDING MULTIPLE OBJECTS}

This section introduces two incremental attribute reduction algorithms on the addition of multiple objects based on knowledge granularity.

4.1. An incremental mechanism to calculate knowledge granularity based on the relation matrix when adding multiple objects

Given a dynamic table, this section introduces the matrix-based incremental mechanisms for computing knowledge granularity when multiple objects are added into a decision table.

Definition 15. Let $S=(U, C \cup D, V, f)$ be a decision table and $U=\left\{x_{1}, x_{2}, \cdots, x_{n}\right\}$. Suppose that $U_{X}=\left\{x_{n+1}, x_{n+2}\right.$, $\left.\cdots, x_{n+t}\right\}$ is the incremental object set, and $R_{C}$ is an equivalence relation on $\left(U \cup U_{X}\right)$. Then the incremental relation matrix $\left(Q_{\left(U \cup U_{X}\right)}^{R_{C}}\right)_{t \times n}=\left(q_{i j}\right)_{t \times n}$ is defined as:

$$
q_{i j}=\left\{\begin{array}{l}
1,\left(u_{(n+i)}, u_{j}\right) \in R_{C} \\
0,\left(u_{(n+i)}, u_{j}\right) \notin R_{C}
\end{array} \quad 1 \leq j \leq n, 1 \leq i \leq t\right.
$$

Definition 16. Let $S=(U, C \cup D, V, f)$ be a decision table and $U=\left\{x_{1}, x_{2}, \cdots, x_{n}\right\}$. Suppose that $U_{X}=\left\{x_{n+1}, x_{n+2}\right.$, $\left.\cdots, x_{n+t}\right\}$ is the incremental object set, and $R_{C}$ is an equivalence relation on $U_{X}$. Then the incremental relation matrix $\left(Z_{U_{X}}^{R_{C}}\right)_{t \times t}=\left(z_{i j}\right)_{t \times t}$ is defined as:

$$
z_{i j}=\left\{\begin{array}{l}
1,\left(u_{(n+i)}, u_{(n+j)}\right) \in R_{C} \\
0,\left(u_{(n+i)}, u_{(n+j)}\right) \notin R_{C}
\end{array} \quad 1 \leq i, j \leq t\right.
$$

Theorem 1. [32]Let $S=(U, C \cup D, V, f)$ be a decision table and $\left(U_{U}^{R_{C}}\right)_{n \times n}$ is the relation matrix. Suppose that $U_{X}=\left\{x_{n+1}, x_{n+2}, \cdots, x_{n+t}\right\}$ is the incremental object set. We can obtain the relation matrices $\left(Q_{\left(U \cup U_{X}\right)}^{R_{C}}\right)_{t \times n}$ and $\left(Z_{U_{X}}^{R_{C}}\right)_{t \times t}$. 
Then,

$$
\left(U_{\left(U \cup U_{X}\right)}^{R_{C}}\right)_{(n+t) \times(n+t)}=\left[\begin{array}{cc}
\left(U_{U}^{R_{C}}\right)_{n \times n} & \left(Q_{\left(U \cup U_{X}\right.}^{R_{C}}\right)_{t \times n}^{T} \\
\left(Q_{\left(U \cup U_{X}\right.}^{R_{C}}\right)_{t \times n} & \left(Z_{U_{X}}^{R_{C}}\right)_{t \times t}
\end{array}\right]
$$

where $\left(Q_{\left(U \cup U_{X}\right)}^{R_{C}}\right)_{t \times n}^{T}$ is the transpose of the relation matrix $\left(Q_{\left(U \cup U_{X}\right)}^{R_{C}}\right)_{t \times n}$.

Theorem 2. Let $S=(U, C \cup D, V, f)$ be a decision table. and $\left(U_{U}^{R_{D}}\right)_{n \times n}$ is the relation matrix. Suppose that $U_{X}=$ $\left\{x_{n+1}, x_{n+2}, \cdots, x_{n+t}\right\}$ is the incremental object set. We can obtain the relation matrices $\left(Q_{\left(U \cup U_{X}\right.}^{R_{D}}\right)_{t \times n}$ and $\left(Z_{U_{X}}^{R_{D}}\right)_{t \times t}$. Then,

$$
\left(U_{U \cup U_{X}}^{R_{D}}\right)_{(n+t) \times(n+t)}=\left[\begin{array}{cc}
\left(U_{U}^{R_{D}}\right)_{n \times n} & \left(Q_{\left(U \cup U_{X}\right)}^{R_{D}}\right)_{t \times n}^{T} \\
\left(Q_{\left(U \cup U_{X}\right)}^{R_{D}}\right)_{t \times n} & \left(Z_{U_{X}}^{R_{D}}\right)_{t \times t}
\end{array}\right]
$$

where $\left(Q_{\left(U \cup U_{X}\right)}^{R_{D}}\right)_{t \times n}^{T}$ is the transpose of the relation matrix $\left(Q_{\left(U \cup U_{X}\right)}^{R_{D}}\right)_{t \times n}$.

Theorem 3. [11]Let $S=(U, C \cup D, V, f)$ be a decision table. $B \subseteq C, B \neq \varnothing, a_{i} \in(C-B), C=\left\{a_{1}, a_{2}, \cdots, a_{n}\right\}$, $\left(M_{U}^{R_{B}}\right)_{n \times n}=\left(b_{i j}\right)_{n \times n}$, and $\left(M_{U}^{R_{\left\{a_{i}\right\}}}\right)_{n \times n}=\left(a_{i j}\right)_{n \times n}$ are the relation matrices. Suppose the attribute $\left\{a_{i}\right\}$ is added to the attribute set $B$. Then, the new relation matrix $\left(M_{U}^{\left.R_{\left(B \cup\left\{a_{i}\right\}\right.}\right)}\right)_{n \times n}=\left(m_{i j}\right)_{n \times n}$, where

$$
m_{i j}=\left\{\begin{array}{l}
a_{i j}, b_{i j} \geq a_{i j} \\
b_{i j}, b_{i j}<a_{i j}
\end{array} \quad 1 \leq i, j \leq n\right.
$$

Theorem 4. Let $S=(U, C \cup D, V, f)$ be a decision table. $U=\left\{x_{1}, x_{2}, \cdots, x_{n}\right\},\left(M_{U}^{R_{C}}\right)_{n \times n},\left(M_{U}^{R_{D}}\right)_{n \times n}$. Suppose that $U_{X}=\left\{x_{n+1}, x_{n+2}, \cdots, x_{n+t}\right\}$ is the incremental object set. We can obtain the relation matrices, $\left(Q_{\left(U \cup U_{X}\right)}^{R_{C}}\right)_{t \times n}$, $\left(Q_{\left(U \cup U_{X}\right)}^{R_{D}}\right)_{t \times n},\left(Q_{\left(U \cup U_{X}\right)}^{R_{(C \cup D)}}\right)_{t \times n},\left(Z_{U_{X}}^{R_{C}}\right)_{t \times t},\left(Z_{U_{X}}^{R_{D}}\right)_{t \times t}$ and $\left(Z_{U_{X}}^{R_{(C \cup D)}}\right)_{t \times t}$. Then,

$$
\begin{aligned}
G P_{\left(U \cup U_{X}\right)}(D \mid C)= & \frac{1}{\left(\left|U \cup U_{X}\right|\right)^{2}}\left(|U|^{2} G P_{U}(D \mid C)+\left|U_{X}\right|^{2} G P_{U_{X}}(D \mid C)+2 S u m\left(\left(Q_{\left(U \cup U_{X}\right)}^{R_{C}}\right)_{t \times n}\right)\right. \\
& -2 S u m\left(\left(Q_{\left(U \cup U_{X}\right)}^{R_{(C \cup D)}}\right)_{t \times n}\right)
\end{aligned}
$$

where $G P_{U}(D \mid C)=\overline{\left(M_{U}^{R_{C}}\right)_{n \times n}}-\overline{\left(M_{U}^{R_{(C \cup D)}}\right)_{n \times n}}$, and $G P_{U_{X}}(D \mid C)=\overline{\left(M_{U_{X}}^{R_{C}}\right)_{t \times t}}-\overline{\left(M_{U_{X}}^{R_{(C \cup D)}}\right)_{t \times t}}$.

Proof. From Definition 11, we have

$$
\begin{aligned}
& G P_{\left(U \cup U_{X}\right)}(D \mid C)=\overline{M_{\left(U \cup U_{X}\right)}^{R_{C}}}-\overline{M_{\left(U \cup U_{X}\right)}^{\left.R_{(}\right)}} \\
= & \frac{1}{\left(\left|U \cup U_{X}\right|\right)^{2}}\left(S u m\left(M_{\left(U \cup U_{X}\right)}^{R_{C}}\right)-S u m\left(M_{\left(U \cup U_{X}\right)}^{R_{(}(\cup \cup)}\right)\right) \\
= & \frac{1}{\left(\left|U \cup U_{X}\right|\right)^{2}}\left(|U|^{2}\left(\overline{M_{U}^{R_{C}}}-\overline{M_{U}^{R_{(C \cup D)}}}\right)+\left|U_{X}\right|^{2}\left(\overline{Z_{U_{X}}^{R_{C}}}-\overline{Z_{U_{X}}^{R_{(C \cup D)}}}\right)+2 S u m\left(\left(Q_{\left(U \cup U_{X}\right)}^{R_{C}}\right)_{t \times n}\right)-2 S u m\left(\left(Q_{\left(U \cup U_{X}\right)}^{R_{(C \cup D)}}\right)_{t \times n}\right)\right)
\end{aligned}
$$

We can get

$G P_{\left(U \cup U_{X}\right)}(D \mid C)=\frac{1}{\left(\left|U \cup U_{X}\right|\right)^{2}}\left(|U|^{2} G P_{U}(D \mid C)+\left|U_{X}\right|^{2} G P_{U_{X}}(D \mid C)+2 S u m\left(\left(Q_{\left(U \cup U_{X}\right)}^{R_{C}}\right)_{t \times n}\right)-2 S u m\left(\left(Q_{\left(U \cup U_{X}\right)}^{R_{(C \cup D)}}\right)_{t \times n}\right)\right)$

Theorem 5. Let $S=(U, C \cup D, V, f)$ be a decision table. $\left(M_{U}^{R_{C}}\right)_{n \times n},\left(M_{U}^{R_{(C-\{a\})}}\right)_{n \times n}$, and $\left(M_{U}^{R_{D}}\right)_{n \times n}$ are the relation matrices, respectively. Suppose that $U_{X}=\left\{x_{n+1}, x_{n+2}, \cdots, x_{n+t}\right\}$ is the incremental object set. We can obtain the 
relation matrices, $\left(Q_{\left(U \cup U_{X}\right)}^{R_{C}}\right)_{t \times n},\left(Q_{\left(U \cup U_{X}\right)}^{R_{(C-\{a)}}\right)_{t \times n}$, and $\left(Z_{U_{X}}^{R_{C}}\right)_{t \times t},\left(Z_{U_{X}}^{R_{(C-\{a\})}}\right)_{t \times t}$. Then, $\forall a \in C$, the new significance (inner significance) of a in $C$ becomes

$$
\begin{aligned}
\operatorname{Sig}_{\left(U \cup U_{X}\right)}^{\text {inner }}(a, C, D)= & \frac{1}{\left(\left|U \cup U_{X}\right|\right)^{2}}\left(|U|^{2} \operatorname{Sig}_{U}^{\text {inner }}(a, C, D)+\left|U_{X}\right|^{2} \operatorname{Sig}_{U_{X}}^{\text {inner }}(a, C, D)+2 S u m\left(\left(Q_{\left(U \cup U_{X}\right)}^{R_{(C-\{a)}}\right)_{t \times n}\right)-\right. \\
& \left.2 \operatorname{Sum}\left(\left(Q_{\left(U \cup U_{X}\right)}^{R_{(C-\{a \backslash D)}}\right)_{t \times n}\right)-2 S u m\left(\left(Q_{\left(U \cup U_{X}\right)}^{R_{C}}\right)_{t \times n}\right)+2 S u m\left(\left(Q_{\left(U \cup U_{X}\right)}^{R_{(C \cup D)}}\right)_{t \times n}\right)\right)
\end{aligned}
$$

where $\operatorname{Sig}_{U}^{\text {inner }}(a, C, D)=\overline{M_{U}^{R_{(C-\{a\})}}}-\overline{M_{U}^{\left.R_{(}(C-\{a\}) \cup D\right)}}-\overline{M_{U}^{R_{C}}}+\overline{M_{U}^{R_{(C \cup D)}}}$, and $\operatorname{Sig}_{U_{X}}^{\text {inner }}(a, C, D)=\overline{Z_{U_{X}}^{R_{(C-\{a)}}}-\overline{Z_{U_{X}}^{R_{((C-\{a\} \cup D)}}}-\overline{Z_{U_{X}}^{R_{C}}}+$ $\overline{Z_{U_{X}}^{R_{(C \cup D)}}}$

Theorem 6. Let $S=(U, C \cup D, V, f)$ be a decision table. $C_{0} \subseteq C,\left(M_{U}^{R_{C_{0}}}\right)_{n \times n},\left(M_{U}^{R_{D}}\right)_{n \times n}$ and $\left(M_{U}^{R_{\left(C_{0} \cup D\right)}}\right)_{n \times n}$ are the relation matrices, respectively. Suppose that $U_{X}=\left\{x_{n+1}, x_{n+2}, \cdots, x_{n+t}\right\}$ is the incremental object set. We can obtain the relation matrices, $\left(Q_{\left(U \cup U_{X}\right)}^{R_{C_{0}}}\right)_{t \times n},\left(Q_{\left(U \cup U_{X}\right)}^{R_{\left(C_{0} \cup D\right)}}\right)_{t \times n}$, and $\left(Z_{U_{X}}^{R_{C_{0}}}\right)_{t \times t},\left(Z_{U_{X}}^{R_{\left(C_{0} \cup(a)\right)}}\right)_{t \times t}$. Then, $\forall a \in\left(C-C_{0}\right)$, the new significance (outer significance) of a in $C_{0}$ becomes

$$
\begin{aligned}
S i g_{\left(U \cup U_{X}\right)}^{\text {outer }}\left(a, C_{0}, D\right)= & \frac{1}{\left(\left|U \cup U_{X}\right|\right)^{2}}\left(|U|^{2} \operatorname{Sig}_{U}^{\text {outer }}\left(a, C_{0}, D\right)+\left|U_{X}\right|^{2} \operatorname{Sig}_{U_{X}}^{\text {outer }}\left(a, C_{0}, D\right)+2 S u m\left(\left(Q_{U \cup U_{X}}^{R_{C_{0}}}\right)_{t \times n}\right)-\right. \\
& \left.2 S u m\left(\left(Q_{\left(U \cup U_{X}\right)}^{R_{\left(C_{0} \cup D\right)}}\right)_{t \times n}\right)-2 S u m\left(\left(Q_{\left(U \cup U_{X}\right)}^{R_{\left(C_{0} \cup(a)\right)}}\right)_{t \times n}\right)+2 S u m\left(\left(Q_{\left(U \cup U_{X}\right)}^{R_{\left(\left(C_{0} \cup(a) \cup D\right)\right.}}\right)_{t \times n}\right)\right)
\end{aligned}
$$

where $S_{i} g_{U}^{\text {outer }}\left(a, C_{0}, D\right)=\overline{M_{U}^{R_{C_{0}}}}-\overline{M_{U}^{R_{\left(C_{0} \cup D\right)}}}-\overline{M_{U}^{R_{\left(C_{0} \cup(a)\right)}}}+\overline{M_{U}^{R_{\left(\left(C_{0} \cup(a) \cup D\right)\right.}}}$, and Sig outer $\left(a, C_{0}, D\right)=\overline{Z_{U_{X}}^{R_{C_{0}}}}-\overline{Z_{U_{X}}^{R_{\left(C_{0} \cup D\right)}}}-\overline{Z_{U_{X}}^{R_{\left(C_{0} \cup(a)\right.}}}+$

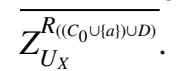

\subsection{A matrix-based incremental reduction algorithm when adding multiple objects}

Based on the incremental mechanisms of relation matrices, this section introduces a matrix-based incremental algorithm (see Algorithm 2) for attribute reduction under knowledge granularity with dynamically varying objects in the decision table.

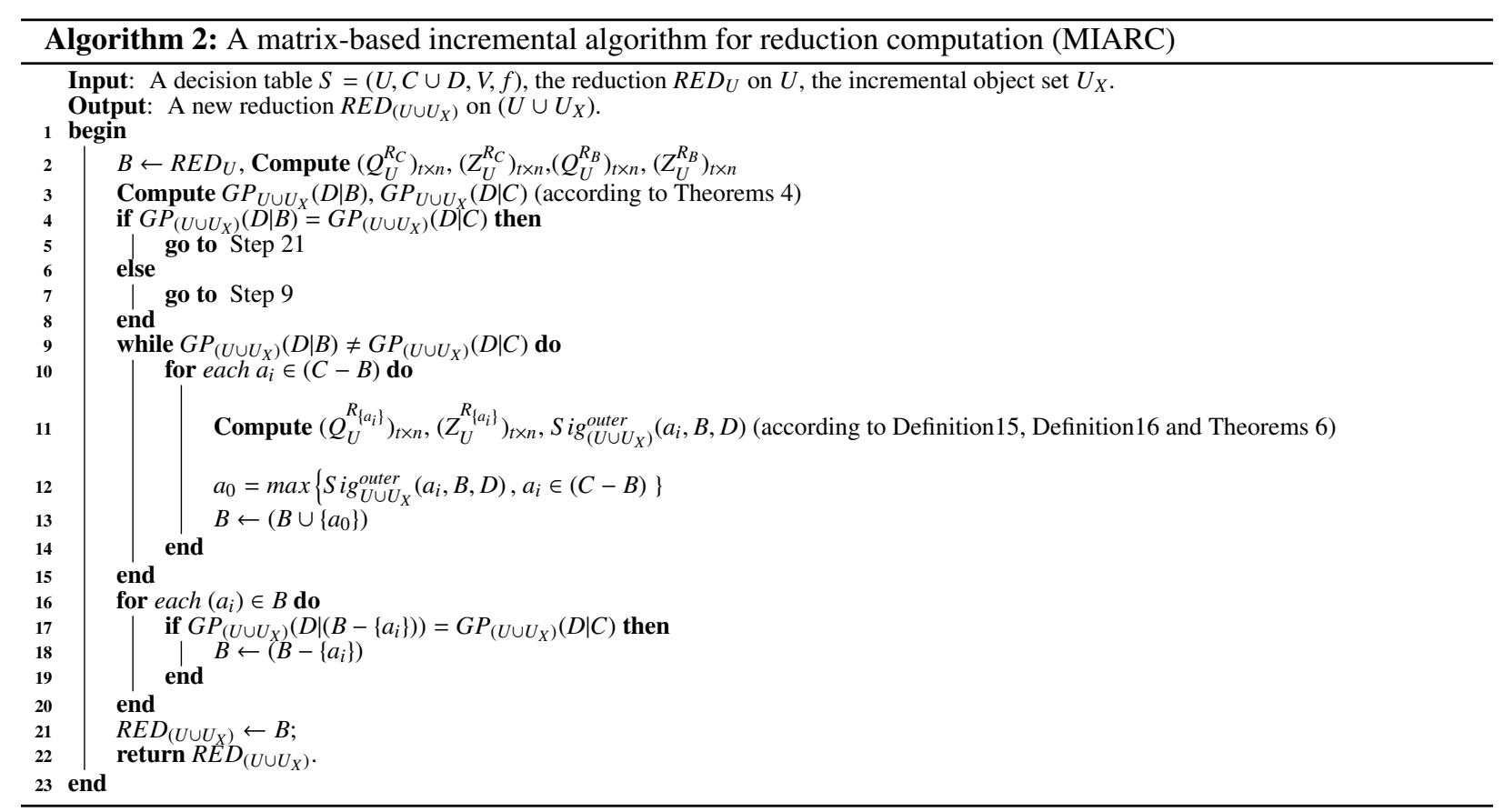


The following is the time complexity analysis of Algorithm 2. When multiple objects are added to the decision table, one can obtain the new value of knowledge granularity by using the incremental mechanism. The time complexity of computing knowledge granularity is $O\left(|C|\left|U_{X}\right|\right)$. The time complexity of Steps 4-8 is $O\left(|C|\left|U_{X}\right|\left|U_{X}\right|+|C|\left|U_{X}\right|^{2}\right)$, the time complexity of Steps 9-14 is $O\left(|C||U|\left|U_{X}\right|+|C|\left|U_{X}\right|^{2}\right)$ and the time complexity of Steps 16-20 is $O\left(|C||U|\left|U_{X}\right|+\right.$ $\left.|C|\left|U_{X}\right|^{2}\right)$. Therefore, the total time complexity of the incremental algorithm is $O\left(|C|\left|U_{X}\right|+|C||U|\left|U_{X}\right|+\left|C \| U_{X}\right|^{2}\right)$ $\approx O\left(|C|\left|U \| U_{X}\right|+|C|\left|U_{X}\right|^{2}\right)$. Table 2 shows a comparison of the time complexity of computing the reduction by Algorithms CAR and MIARC.

Table 2: A Comparison of Time Complexity of CAR and MIARC.

\begin{tabular}{ccc}
\hline Reduction Algorithms & $C A R$ & MIARC \\
\hline Time Complexity & $O\left(|C||U|\left|U_{X}\right|+|C||U|^{2}+|C|\left|U_{X}\right|^{2}\right)$ & $O\left(\left|C\left\|\left|U \| U_{X}\right|+|C|\left|U_{X}\right|^{2}\right)\right.\right.$ \\
\hline
\end{tabular}

From Table 2, because $O\left(\left.|C||U \|| U_{X}|+| C|| U_{X}\right|^{2}\right)$ is much lower than $O\left(\left|C\left\|\left|U \| U_{X}\right|+|C||U|^{2}+|C|\left|U_{X}\right|^{2}\right)\right.\right.$, we can conclude the time complexity of computing the reduction by the matrix-based incremental algorithm is usually much smaller than that of the non-incremental algorithm. Hence, the incremental algorithm MIARC can save more computational time than CAR.

\subsection{An incremental mechanism to calculate knowledge granularity based on non-matrix after adding multiple objects}

When data sets are smaller, the matrix-based methods for updating the reduction is efficient. However, for the larger data sets, matrix-based incremental methods for computing the reduction under knowledge granularity are inefficient since they need more computational space and memory. To overcome the deficiency, an updated incremental algorithm is developed to deal with such data sets. This subsection introduces incremental mechanisms for computing knowledge granularity.

For the sake of convenience, we give some explanations that will be applied in the following theorems. Let $S=(U, C \cup D, V, f)$ be a decision table. $B \subseteq C, U / B=\left\{X_{1}, X_{2}, \cdots, X_{j}\right\}$. It is assumed that $U_{X}$ is the incremental object set, $U_{X} / B=\left\{Y_{1}, Y_{2}, \cdots, Y_{j^{\prime}}\right\}$. There may be some conditional classes with the same attribute values on $B$ between $U / B$ and $U_{X} / B$. We assume that $\left(U \cup U_{X}\right) / B=\left\{X_{1}^{\prime}, X_{2}^{\prime}, \cdots, X_{k}^{\prime}, X_{k+1}, X_{k+2}, \cdots, X_{j}, Y_{k+1}, Y_{k+2}, \cdots, Y_{j^{\prime}}\right\}$. $X_{i}^{\prime}=X_{i} \cup Y_{i}(i=1,2, \cdots, k)$ in $\left(U \cup U_{X}\right) / B$ denote these conditional classes which can be combined, that is to say, the attribute values of $X_{i} \in U / B$ and $Y_{i} \in U_{X} / B$ are the same. $X_{i} \in U / B(i=k+1, k+2, \cdots, j)$ and $Y_{j} \in U_{X} / B(j=$ $\left.k+1, k+2, \cdots, j^{\prime}\right)$ denote these conditional classes which can not be combined, respectively [21].

Example 3. In Table $1, U=\{1,2,3,4,5,6,7,8,9\}$ and $U / C=\{\{1\},\{2,4\},\{3,5\},\{6,7\},\{8,9\}\}$. Let the incremental object set $U_{X}=\{10,11,12,13\}$. Then $U_{X} / C=\{\{10,12\},\{11\},\{13\}\}$. Suppose that the attribute values of $\{11\}$ is equal to that of $\{6,7\}$ and the attribute values of $\{13\}$ is equal to that of $\{1\}$. Then, we can get $X_{1}^{\prime}=\{6,7,11\}, X_{2}^{\prime}=$ $\{1,13\}, X_{3}=\{2,4\}, X_{4}=\{3,5\}, X_{5}=\{8,9\}, Y_{2}=\{10,12\}$. Thus $\left(U \cup U_{X}\right) / C=\{\{6,7,11\},\{1,13\},\{2,4\},\{3,5\},\{8,9\}$, $\{10,12\}\}$. Obviously, $j=5, j^{\prime}=3, k=2$.

Given a decision table, incremental mechanisms based on knowledge granularity are introduced in the following theorems.

Theorem 7. Let $S=(U, C \cup D, V, f)$ be a decision table. $U / C=\left\{X_{1}, X_{2}, \cdots, X_{m}\right\}$. The knowledge granularity of $C$ is $G P_{U}(C)$. Suppose that $U_{X}$ is the incremental object set and $U_{X} / C=\left\{Y_{1}, Y_{2}, \cdots, Y_{m^{\prime}}\right\}$. We denote $\left(U \cup U_{X}\right) / C=\left\{X_{1}^{\prime}, X_{2}^{\prime}, \cdots, X_{k}^{\prime}, X_{k+1}, X_{k+2}, \cdots, X_{m}, Y_{k+1}, Y_{k+2}, \cdots, Y_{m^{\prime}}\right\}$. Then,

$$
G P_{\left(U \cup U_{X}\right)}(C)=\frac{1}{\left(\left|U \cup U_{X}\right|\right)^{2}}\left(|U|^{2} G P_{U}(C)+\left|U_{X}\right|^{2} G P_{U_{X}}(C)+2 \sum_{i=1}^{k}\left|X_{i}\right|\left|Y_{i}\right|\right)
$$

Proof. From Definition 4, we have

$$
G P_{\left(U \cup U_{X}\right)}(C)=\sum_{i=1}^{k} \frac{\left|X_{i}^{\prime}\right|^{2}}{\left(\left|U \cup U_{X}\right|\right)^{2}}+\sum_{i=k+1}^{m} \frac{\left|X_{i}\right|^{2}}{\left(\left|U \cup U_{X}\right|\right)^{2}}+\sum_{i=k+1}^{m^{\prime}} \frac{\left|Y_{i}\right|^{2}}{\left(\left|U \cup U_{X}\right|\right)^{2}}
$$


Since $X_{i}^{\prime}=X_{i} \cup Y_{i}(i=1,2, \cdots, k)$, we have

$$
\begin{gathered}
G P_{\left(U \cup U_{X}\right)}(C)=\sum_{i=1}^{k} \frac{\left(\left|X_{i}\right|+\left|Y_{i}\right|\right)^{2}}{\left(\left|U \cup U_{X}\right|\right)^{2}}+\sum_{i=k+1}^{m} \frac{\left|X_{i}\right|^{2}}{\left(\left|U \cup U_{X}\right|\right)^{2}}+\sum_{i=k+1}^{m^{\prime}} \frac{\left|Y_{i}\right|^{2}}{\left(\left|U \cup U_{X}\right|\right)^{2}} \\
=\frac{1}{\left(\left|U \cup U_{X}\right|\right)^{2}}\left(\sum_{i=1}^{k}\left(\left|X_{i}\right|+\left|Y_{i}\right|\right)^{2}+\sum_{i=k+1}^{m}\left|X_{i}\right|^{2}+\sum_{i=k+1}^{m^{\prime}}\left|Y_{i}\right|^{2}\right)
\end{gathered}
$$

Since $\left(\left|X_{i}\right|+\left|Y_{i}\right|\right)^{2}=\left|X_{i}\right|^{2}+\left|Y_{i}\right|^{2}+2\left|X_{i}\right|\left|Y_{i}\right|$, we can get

$$
=\frac{1}{\left(\left|U \cup U_{X}\right|\right)^{2}}\left(|U|^{2}\left(\sum_{i=1}^{k} \frac{\left|X_{i}\right|^{2}}{|U|^{2}}+\sum_{i=k+1}^{m} \frac{\left|X_{i}\right|^{2}}{|U|^{2}}\right)+\left|U_{X}\right|^{2}\left(\sum_{i=1}^{k} \frac{\left|Y_{i}\right|^{2}}{\left|U_{X}\right|^{2}}+\sum_{i=k+1}^{m^{\prime}} \frac{\left|Y_{i}\right|^{2}}{\left|U_{X}\right|^{2}}\right)+2 \sum_{i=1}^{k}\left|X_{i} \| Y_{i}\right|\right.
$$

Then

$$
G P_{\left(U \cup U_{X}\right)}(C)=\frac{1}{\left(\left|U \cup U_{X}\right|\right)^{2}}\left(|U|^{2} G P_{U}(C)+\left|U_{X}\right|^{2} G P_{U_{X}}(C)+2 \sum_{i=1}^{k}\left|X_{i}\right|\left|Y_{i}\right|\right)
$$

Theorem 8. Let $S=(U, C \cup D, V, f)$ be a decision table. $U /(C \cup D)=\left\{M_{1}, M_{2}, \cdots, M_{n}\right\}$. The knowledge granularity of $(C \cup D)$ is $G P_{U}(C \cup D)$. Suppose that $U_{X}$ is the incremental object set and $U_{X} /(C \cup D)=\left\{N_{1}, N_{2}, \cdots, N_{n^{\prime}}\right\}$. We denote $\left(U \cup U_{X}\right) /(C \cup D)=\left\{M_{1}^{\prime}, M_{2}^{\prime}, \cdots, M_{k}^{\prime}, M_{k+1}, M_{k+2}, \cdots, M_{n}, N_{k+1}, N_{k+2}, \cdots, N_{n^{\prime}}\right\}$. Then,

$$
G P_{\left(U \cup U_{X}\right)}(C \cup D)=\frac{1}{\left(\left|U \cup U_{X}\right|\right)^{2}}\left(|U|^{2} G P_{U}(C \cup D)+\left|U_{X}\right|^{2} G P_{U_{X}}(C \cup D)+2 \sum_{i=1}^{k}\left|M_{i}\right|\left|N_{i}\right|\right)
$$

Proof. The proof is similar to that of Theorem 7.

Theorem 9. Let $S=(U, C \cup D, V, f)$ be a decision table. $U / C=\left\{X_{1}, X_{2}, \cdots, X_{m}\right\}$ and $U /(C \cup D)=\left\{M_{1}, M_{2}, \cdots\right.$, $\left.M_{n}\right\}$. The knowledge granularity of $D$ with respect to $C$ is $G P_{U}(D \mid C)$. Suppose that $U_{X}$ is the incremental object set, $U_{X} / C=\left\{Y_{1}, Y_{2}, \cdots, Y_{m^{\prime}}\right\}$ and $U_{X} /(C \cup D)=\left\{N_{1}, N_{2}, \cdots, N_{n^{\prime}}\right\}$. We denote $\left(U \cup U_{X}\right) / C=\left\{X_{1}^{\prime}, X_{2}^{\prime}, \cdots, X_{k}^{\prime}, X_{k+1}, X_{k+2}\right.$, $\left.\cdots, X_{m}, Y_{k+1}, Y_{k+2}, \cdots, Y_{m^{\prime}}\right\}$ and $\left(U \cup U_{X}\right) /(C \cup D)=\left\{M_{1}^{\prime}, M_{2}^{\prime}, \cdots, M_{k}^{\prime}, M_{k+1}, M_{k+2}, \cdots, M_{n}, N_{k+1}, N_{k+2}, \cdots, N_{n^{\prime}}\right\}$. Then,

$$
G P_{\left(U \cup U_{X}\right)}(D \mid C)=\frac{1}{\left(\left|U \cup U_{X}\right|\right)^{2}}\left(|U|^{2} G P_{U}(D \mid C)+\left|U_{X}\right|^{2} G P_{U_{X}}(D \mid C)+2 \sum_{i=1}^{k}\left|X_{i}\right|\left|Y_{i}\right|-2 \sum_{i=1}^{k}\left|M_{i}\right|\left|N_{i}\right|\right)
$$

Proof. From Definition 5, we have

$$
G P_{\left(U \cup U_{X}\right)}(D \mid C)=G P_{\left(U \cup U_{X}\right)}(C)-G P_{\left(U \cup U_{X}\right)}(C \cup D)
$$

Since

$$
G P_{\left(U \cup U_{X}\right)}(C)=\frac{1}{\left(\left|U \cup U_{X}\right|\right)^{2}}\left(|U|^{2} G P_{U}(C)+\left|U_{X}\right|^{2} G P_{U_{X}}(C)+2 \sum_{i=1}^{k}\left|X_{i}\right|\left|Y_{i}\right|\right)
$$

and

$$
G P_{\left(U \cup U_{X}\right)}(C \cup D)=\frac{1}{\left(\left|U \cup U_{X}\right|\right)^{2}}\left(|U|^{2} G P_{U}(C \cup D)+\left|U_{X}\right|^{2} G P_{U_{X}}(C \cup D)+2 \sum_{i=1}^{k}\left|M_{i}\right|\left|N_{i}\right|\right)
$$

Then we have

$$
G P_{\left(U \cup U_{X}\right)}(D \mid C)=\frac{1}{\left(\left|U \cup U_{X}\right|\right)^{2}}\left(|U|^{2} G P_{U}(D \mid C)+\left|U_{X}\right|^{2} G P_{U_{X}}(D \mid C)+2 \sum_{i=1}^{k}\left|X_{i}\right|\left|Y_{i}\right|-2 \sum_{i=1}^{k}\left|M_{i}\right|\left|N_{i}\right|\right)
$$

In the following, the incremental mechanisms of the attribute significance measure (inner and outer significance) are introduced in Theorems 10 and 11 . 
Theorem 10. Let $S=(U, C \cup D, V, f)$ be a decision table. $U / C=\left\{X_{1}, X_{2}, \cdots, X_{m}\right\}, U /(C \cup D)=\left\{M_{1}, M_{2}, \cdots, M_{n}\right\}$ , $U /(C-\{a\})=\left\{E_{1}, E_{2}, \cdots, E_{b}\right\}$ and $U /((C-\{a\}) \cup D)=\left\{Z_{1}, Z_{2}, \cdots, Z_{b^{\prime}}\right\}$. Inner significance of $a$ in $C$ is $S$ ig ${ }_{U}^{\text {inner }}(a, C, D)$. Suppose that $U_{X}$ is the incremental object set, $U_{X} / C=\left\{Y_{1}, Y_{2}, \cdots, Y_{m^{\prime}}\right\}, U_{X} /(C \cup D)=\left\{N_{1}, N_{2}, \cdots\right.$, $\left.N_{n^{\prime}}\right\}, U_{X} /(C-\{a\})=\left\{F_{1}, F_{2}, \cdots, F_{c}\right\}$ and $U_{X} /((C-\{a\}) \cup D)=\left\{L_{1}, L_{2}, \cdots, L_{c^{\prime}}\right\}$. We denote $\left(U \cup U_{X}\right) / C=$ $\left\{X_{1}^{\prime}, X_{2}^{\prime}, \cdots, X_{k}^{\prime}, X_{k+1}, X_{k+2}, \cdots, X_{m}, Y_{k+1}, Y_{k+2}, \cdots, Y_{m^{\prime}}\right\},\left(U \cup U_{X}\right) /(C \cup D)=\left\{M_{1}^{\prime}, M_{2}^{\prime}, \cdots, M_{k}^{\prime}, M_{k+1}, M_{k+2}, \cdots, M_{n}\right.$, $\left.N_{k+1}, N_{k+2}, \cdots, N_{n^{\prime}}\right\},\left(U \cup U_{X}\right) /(C-\{a\})=\left\{E_{1}^{\prime}, E_{2}^{\prime}, \cdots, E_{k}^{\prime}, E_{k+1}, E_{k+2}, \cdots, E_{b}, F_{k+1}, F_{k+2}, \cdots, F_{c}\right\}$ and $U \cup U_{X} /((C-$ $\{a\}) \cup D)=\left\{Z_{1}^{\prime}, Z_{2}^{\prime}, \cdots, Z_{k}^{\prime}, Z_{k+1}, Z_{k+2}, \cdots, Z_{b^{\prime}}, L_{k+1}, L_{k+2}, \cdots, L_{c^{\prime}}\right\}$. Then, $\forall a \in C$,

$$
\begin{aligned}
\operatorname{Sig}_{\left(U \cup U_{X}\right)}^{\text {inner }}(a, C, D) & =\frac{1}{\left(\left|U \cup U_{X}\right|\right)^{2}}\left(|U|^{2} \operatorname{Sig}_{U}^{\text {inner }}(a, C, D)+\left|U_{X}\right|^{2} \operatorname{Sig}_{U_{X}}^{\text {inner }}(a, C, D)+2 \sum_{i=1}^{k}\left|E_{i}\right|\left|F_{i}\right|-2 \sum_{i=1}^{k}\left|Z_{i}\right|\left|L_{i}\right|\right. \\
& \left.-2 \sum_{i=1}^{k}\left|X_{i}\left\|Y_{i}\left|+2 \sum_{i=1}^{k}\right| M_{i}\right\| N_{i}\right|\right)
\end{aligned}
$$

Proof. The proof is similar to that of Theorem 9.

Theorem 11. Let $S=(U, C \cup D, V, f)$ be a decision table. $C_{0} \subseteq C, U / C_{0}=\left\{Q_{1}, Q_{2}, \cdots, Q_{d}\right\}, U /\left(C_{0} \cup D\right)=$ $\left\{G_{1}, G_{2}, \cdots, G_{d^{\prime}}\right\}, U /\left(C_{0} \cup\{a\}\right)=\left\{H_{1}, H_{2}, \cdots, H_{z}\right\}$ and $U /\left(\left(C_{0} \cup\{a\}\right) \cup D\right)=\left\{P_{1}, P_{2}, \cdots, P_{z^{\prime}}\right\}$. Outer significance of $a$ in $C_{0}$ is $S$ ig ${ }_{U}^{\text {outer }}\left(a, C_{0}, D\right)$. Suppose that $U_{X}$ is the incremental object set, $U_{X} / C_{0}=\left\{O_{1}, O_{2}, \cdots, O_{w}\right\}$, $U_{X} /\left(C_{0} \cup D\right)=\left\{T_{1}, T_{2}, \cdots, T_{w^{\prime}}\right\}, U_{X} /\left(C_{0} \cup\{a\}\right)=\left\{W_{1}, W_{2}, \cdots, W_{i}\right\}$ and $U_{X} /\left(\left(C_{0} \cup\{a\}\right) \cup D\right)=\left\{I_{1}, I_{2}, \cdots\right.$, $\left.I_{l^{\prime}}\right\}$. We denote $\left(U \cup U_{X}\right) / C_{0}=\left\{Q_{1}^{\prime}, Q_{2}^{\prime}, \cdots, Q_{k}^{\prime}, \quad Q_{k+1}, Q_{k+2}, \cdots, Q_{d}, O_{k+1}, O_{k+2}, \cdots, O_{w}\right\}, U \cup U_{X} /\left(C_{0} \cup D\right)=$ $\left\{G_{1}^{\prime}, G_{2}^{\prime}, \cdots, G_{k}^{\prime}, G_{k+1}, G_{k+2}, \cdots, G_{d^{\prime}}, T_{k+1}, T_{k+2}, \cdots, T_{w^{\prime}}\right\},\left(U \cup U_{X}\right) /\left(C_{0} \cup\{a\}\right)=\left\{H_{1}^{\prime}, H_{2}^{\prime}, \cdots, H_{k}^{\prime}, H_{k+1}, H_{k+2}, \cdots, H_{z}\right.$, $\left.W_{k+1}, W_{k+2}, \cdots, W_{i}\right\}$, and $\left(U \cup U_{X}\right) /\left(\left(C_{0} \cup\{a\}\right) \cup D\right)=\left\{P_{1}^{\prime}, P_{2}^{\prime}, \cdots, P_{k}^{\prime} P_{k+1}, P_{k+2}, \cdots, P_{z^{\prime}}, I_{k+1}, I_{k+2}, \cdots, I_{l^{\prime}}\right\}$. Then, $\forall a \in\left(C-C_{0}\right)$,

$$
\begin{aligned}
\operatorname{Sig}_{\left(U \cup U_{X}\right)}^{\text {outer }}\left(a, C_{0}, D\right)= & \frac{1}{\left(\left|U \cup U_{X}\right|\right)^{2}}\left(|U|^{2} \operatorname{Sig}_{U}^{\text {outer }}\left(a, C_{0}, D\right)+\left|U_{X}\right|^{2} \operatorname{Sig}_{U_{X}}^{\text {outer }}\left(a, C_{0}, D\right)+2 \sum_{i=1}^{k}\left|Q_{i}\right|\left|O_{i}\right|\right. \\
& \left.-2 \sum_{i=1}^{k}\left|G_{i}\right|\left|T_{i}\right|-2 \sum_{i=1}^{k}\left|H_{i}\right|\left|W_{i}\right|+2 \sum_{i=1}^{k}\left|P_{i}\right|\left|I_{i}\right|\right)
\end{aligned}
$$

Proof. The proof is similar to that of Theorem 9.

\subsection{An incremental reduction algorithm based on non-matrix when adding multiple objects}

Based on the incremental mechanisms of knowledge granularity, this subsection introduces an incremental algorithm (see Algorithm 3) based on non-matrix for attribute reduction under knowledge granularity with dynamically varying objects in the decision table.

The following is the time complexity analysis of Algorithm 3. When multiple objects are added to the decision table, one can obtain the new value of knowledge granularity by using the incremental mechanism. The time complexity of computing knowledge granularity is $O\left(|C|\left|U_{X}\right|\right)$. The time complexity of Steps 4-9 is $O\left(|C|\left|U_{X}\right|^{2}\right)$, the time complexity of Steps 10-15 is $O\left(\left|C\|m\| m^{\prime}\right|\right)$, where the specific description of $m, m^{\prime}$ is listed in Theorem 7, and the time complexity of Steps 17-21 is $O\left(\left|C\|m\| m^{\prime}\right|\right)$. Therefore, the total time complexity of the incremental algorithm is $O\left(|C|\left|U_{X}\right|+\left|C\|m\| m^{\prime}\right|+|C|\left|U_{X}\right|^{2}\right) \approx O\left(\left|C\|m\| m^{\prime}\right|+|C|\left|U_{X}\right|^{2}\right)$. Table 3 shows a comparison of the time complexity of computing the reduction by Algorithms MICAR and IARC.

From Table 3 , since $|m|\left|m^{\prime}\right|$ is usually much smaller than $|U|\left|U_{X}\right|$, we can conclude that the time complexity of computing the reduction by incremental algorithm based on non-matrix is usually much smaller than that of matrixbased incremental algorithm. Therefore, the incremental algorithm IARC is more efficient than algorithm MICAR.

Example 4. Computing new reduction based on knowledge granularity by using Algorithm 3.

For Table 1, its reduction obtained by using Algorithm 1 based on knowledge granularity is $B=\{b, f\}$. Suppose that $U_{X}=\{10,11,12\}$ is the added object set. $10=\{1,0,0,0,1,0\}, 11=\{0,0,1,0,1,1\}, 12=\{0,1,1,1,1,1\}$. 


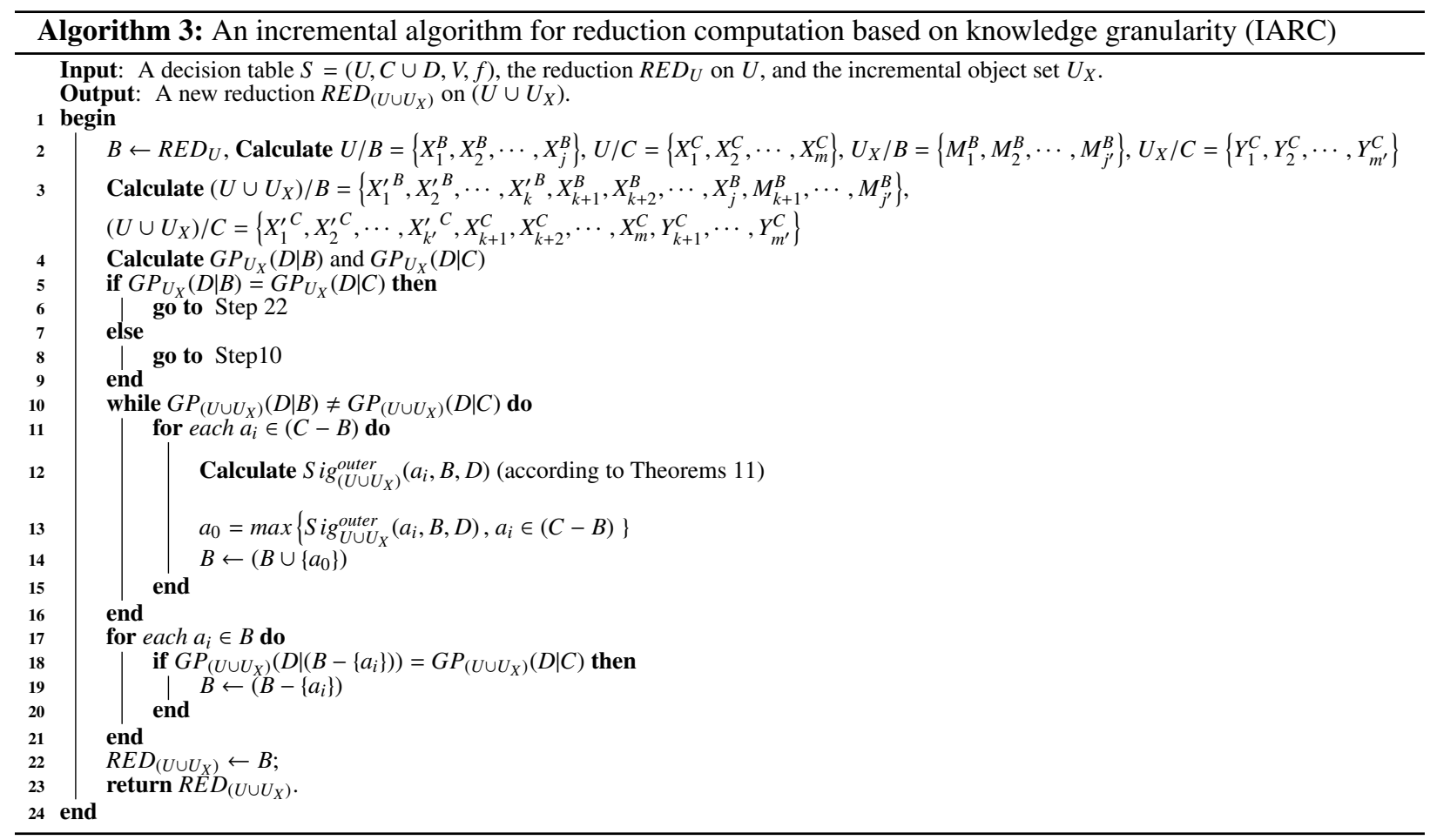

Table 3: A Comparison of Time Complexity of MICAR and IARC.

\begin{tabular}{ccc}
\hline Reduction Algorithms & $M I C A R$ & $I A R C$ \\
\hline Time Complexity & $O\left(|C|\left|U \| U_{X}\right|+|C|\left|U_{X}\right|^{2}\right)$ & $O\left(\left|C\left\|\left|m \| m^{\prime}\right|+|C|\left|U_{X}\right|^{2}\right)\right.\right.$ \\
\hline
\end{tabular}

Based on Step $2, B=\{b, f\},\left(U \cup U_{X}\right) / C=\{\{1,10\},\{2,4,11\},\{3,5\},\{6,7\},\{8,9\},\{12\}\}$,

$\left(U \cup U_{X}\right) / B=\{\{1,10,2,4,11\},\{8,, 9,12\},\{3,5\},\{6,7\}\}$.

Based on Steps $4-9$, we get $G P_{U_{X}}(D \mid B)=\frac{1}{9}$ and $G P_{U_{X}}(D \mid C)=0$. Hence, this example requires to add condition attributes from $(C-B)$.

According to Steps 10-16, we add condition attributes a, e to B. Thus, $B=\{b, f, a, e\}$. Now, we have $G P_{\left(U \cup U_{X}\right)}(D \mid B)=$ $G P_{\left(U \cup U_{X}\right)}(D \mid C)=\frac{3}{72}$. Then go to Step 17 .

Based on Steps 17-20, there is not any attribute in $B$ which needs to be removed and the final reduction is $R E D_{\left(U \cup U_{X}\right)}=\{b, f, a, e\}$.

\section{Experimental evaluations}

\subsection{A description of datasets and experimental environment}

In this subsection, we perform a series of experiments to show the effectiveness and efficiency of the proposed two incremental algorithms for attribute reduction when multiple objects are added to the decision table. In the experiments, the data sets downloaded from UCI are listed in Table 4. All experiments have been run on a computer with 2.66GHz CPU Inter Core2 Quad Q8200 and 4.0 GB of memory and 32-bit Windows 7 operation system. The proposed algorithms are written in C\# language.

\subsection{A comparison of running time on different data sets}

In this subsection, we compare the computation time between the non-incremental algorithm and the proposed two incremental algorithms on the 9 different data sets shown in Table 4. In these data sets, since Mushroom and 
Table 4: A description of data sets

\begin{tabular}{|c|c|c|c|c|c|}
\hline & Data sets & Samples & Attributes & Classes & Source \\
\hline 1 & Breast-cancer-wisconsin & 683 & 9 & 2 & UCI \\
\hline 2 & Tic-tea-toe & 958 & 9 & 2 & UCI \\
\hline 3 & Kr-vs-kp & 3196 & 36 & 2 & UCI \\
\hline 4 & Krkopt & 28056 & 6 & 18 & UCI \\
\hline 5 & Letter-recognition(Letter) & 20000 & 16 & 26 & UCI \\
\hline 6 & shuttle & 43500 & 9 & 7 & UCI \\
\hline 7 & Mushroom & 5644 & 22 & 2 & UCI \\
\hline 8 & Dermatology & 366 & 34 & 6 & UCI \\
\hline 9 & Backup-large & 307 & 35 & 19 & UCI \\
\hline
\end{tabular}

Dermatology have missing values, then we simply remove the objects with missing values since the proposed algorithms cannot deal with missing values. In order to distinguish the computational efficiency with respect to the data sets in the increasing number of objects, 50\% objects of each data set in Table 4 are selected as the basic object set, and the remaining $50 \%$ objects are selected as new object set. When new objects are added to the basic data set, we use non-incremental algorithm and incremental algorithms to update the reduction of each data set. The experimental results are listed in Tables 5 and 6 . These tables show the number of selected features (NSF), evaluational results of found reductions and computation time of each employed data set between the non-incremental and incremental algorithms for attribute reduction when adding objects.

According to the experimental results, in each data set, the number of selected features and attribute reduction generated by the non-incremental CAR and incremental algorithms MIARC, IARC are relatively close. However, the computational time of incremental algorithms MIARC, IARC is much smaller than that of the non-incremental algorithm CAR, especially for the larger data sets, e.g., Shuttle, Letter and Mushroom. In addition, from Tables 5 and 6, attribute reduction generated by the incremental algorithm MIARC is identical to that of IARC. However, the computational time of incremental algorithm IARC is smaller than that of incremental algorithm MIARC. Hence, The experimental results indicate that incremental algorithm IARC is efficient to process the large data set with dynamically varying objects.

Table 5: A Comparison of CAR and MIARC on Computational Time.

\begin{tabular}{|c|c|c|c|c|c|c|}
\hline \multirow{2}{*}{ Data sets } & \multicolumn{3}{|c|}{ CAR } & \multicolumn{3}{|c|}{ MIARC } \\
\hline & NSF & Reduction & Times/s & NSF & Reduction & Times/s \\
\hline cancer & 5 & $6,3,2,5,1$ & 0.857 & 5 & $6,3,2,5,1$ & 0.134 \\
\hline Tic-tea-toe & 8 & $1,5,4,2,9,7,3,8$ & 1.407 & 8 & $1,2,5,4,9,7,3,8$ & 1.322 \\
\hline Kr-vs-kp & 30 & $\begin{array}{c}1,3,4,5,6,7,10,12,13,15,16,17,18,20,21,23,24 \\
25,26,27,28,30,31,33,34,35,36,11,32,22\end{array}$ & 35.87 & 31 & $\begin{array}{c}1,3,4,5,6,7,10,12,13,14,16,17,21,23,26,30,31,33 \\
34,35,36,2,18,24,9,22,11,20,25,27,28\end{array}$ & 15.55 \\
\hline Mushroom & 9 & $1,5,20,9,3,13,12,15,10$ & 131.6 & 7 & $1,5,8,20,10,18,3$ & 86.64 \\
\hline Dermatology & 6 & $1,34,16,4,19,28$ & 0.646 & 7 & $1,34,16,4,3,28,19$ & 0.025 \\
\hline Backup-large & 11 & $7,16,1,22,10,29,6,8,21,4,15$ & 0.484 & 17 & $1,22,10,7,13,6,15,29,4,3,8,9,2,11,30,31,16$ & 0.248 \\
\hline
\end{tabular}

\subsection{A comparison of running time on data sets with different numbers of objects}

In this subsection, we select data sets from Table 4 to compare the non-incremental algorithm and the incremental algorithms based on knowledge granularity with different numbers of objects of each data set. We select $50 \%$ objects of each data set in Table 4 as the basic object sets, then select 20\%, 40\%, , , 100\% from the remaining 50\% objects, in order, as incremental object sets [13]. The combination of each incremental object sets and the basic object sets is regarded as the new data sets. We use these new data sets to calculate the computational time by non-incremental and incremental methods, respectively. By comparing computational time of non-incremental and incremental algorithms, we demonstrate the efficiency and effectiveness of the proposed incremental algorithms. Experimental results of these data sets are outlined in Tables 7 and 8. More detailed change trend lines of each of two methods with the increasing 
Table 6: A Comparison of CAR and IARC on Computational Time.

\begin{tabular}{|c|c|c|c|c|c|c|}
\hline \multirow{2}{*}{ Data sets } & \multicolumn{3}{|c|}{ CAR } & \multicolumn{3}{|c|}{ IARC } \\
\hline & $\mathrm{NSF}$ & Reduction & Times/s & NSF & Reduction & Times/s \\
\hline cancer & 5 & $6,3,2,5,1$ & 0.121 & 5 & $6,3,2,5,1$ & 0.022 \\
\hline Tic-tea-toe & 8 & $1,5,4,2,9,7,3,8$ & 0.588 & 8 & $1,2,5,4,9,7,3,8$ & 0.186 \\
\hline Kr-vs-kp & 30 & $\begin{array}{c}1,3,4,5,6,7,10,12,13,15,16,17,18,20,21,23,24, \\
25,26,27,28,30,31,33,34,35,36,11,32,22\end{array}$ & 39.32 & 31 & $\begin{array}{c}1,3,4,5,6,7,10,12,13,14,16,17,21,23,26,30,31,33 \\
34,35,36,2,18,24,9,22,11,20,25,27,28\end{array}$ & 17.93 \\
\hline Krkopt & 6 & $1,2,3,4,5,6$ & 92.11 & 6 & $1,2,3,4,5,6$ & 35.72 \\
\hline Letter & 13 & $4,8,15,9,11,13,10,7,6,12,14,3,5$ & 376.1 & 14 & $8,2,15,9,11,4,5,3,13,10,1,7,6,12$ & 36.24 \\
\hline shuttle & 4 & $2,9,8,1$ & 610.8 & 4 & $2,9,8,1$ & 105.7 \\
\hline
\end{tabular}

size of data sets are displayed in Figs. 1-2, respectively. In each sub-figure of Figs. 1-2, $\mathrm{x}$-axis is the size of data sets and $y$-axis is the time for computing reductions. Dot lines depict the computational time of non-incremental reduction algorithms and square lines show the computational time of incremental reduction algorithms.

Table 7: A Comparison of CAR and MIARC on Different Numbers of Objects.

\begin{tabular}{ccccccccccc}
\hline \multirow{2}{*}{ Data sets } & \multicolumn{3}{c}{ CAR } & \multicolumn{3}{c}{ MIARC } \\
\cline { 2 - 9 } & $20 \%$ & $40 \%$ & $60 \%$ & $80 \%$ & $100 \%$ & $20 \%$ & $40 \%$ & $60 \%$ & $80 \%$ & $100 \%$ \\
\hline cancer & 0.449 & 0.498 & 0.586 & 0.716 & 0.857 & 0.098 & 0.101 & 0.12 & 0.131 & 0.134 \\
Tic-tea-toe & 0.528 & 0.795 & 0.986 & 1.202 & 1.407 & 0.114 & 0.122 & 0.134 & 1.157 & 1.322 \\
Dermatology & 0.258 & 0.342 & 0.439 & 0.585 & 0.646 & 0.012 & 0.014 & 0.018 & 0.022 & 0.025 \\
Backup-large & 0.202 & 0.252 & 0.307 & 0.398 & 0.484 & 0.009 & 0.013 & 0.155 & 0.203 & 0.248 \\
Kr-vs-kp & 19.27 & 24.19 & 29.74 & 30.81 & 35.87 & 0.761 & 1.081 & 1.485 & 1.882 & 15.55 \\
Mushroom & 52.54 & 66.77 & 83.87 & 104.5 & 131.6 & 1.912 & 2.633 & 3.359 & 72.27 & 86.64 \\
\hline
\end{tabular}

Table 8: A Comparison of CAR and IARC on Different Numbers of Objects.

\begin{tabular}{ccccccccccc}
\hline \multirow{2}{*}{ Data sets } & \multicolumn{3}{c}{ CAR } & \multicolumn{3}{c}{ IARC } \\
\cline { 2 - 9 } & $20 \%$ & $40 \%$ & $60 \%$ & $80 \%$ & $100 \%$ & $20 \%$ & $40 \%$ & $60 \%$ & $80 \%$ & $100 \%$ \\
\hline cancer & 0.061 & 0.075 & 0.093 & 0.103 & 0.121 & 0.01 & 0.016 & 0.017 & 0.018 & 0.022 \\
Tic-tea-toe & 0.17 & 0.318 & 0.367 & 0.438 & 0.478 & 0.024 & 0.074 & 0.127 & 0.153 & 0.186 \\
Kr-vs-kp & 11.25 & 14.77 & 19.07 & 32.02 & 39.32 & 0.519 & 0.978 & 1.19 & 12.82 & 17.93 \\
Krkopt & 36.89 & 49.87 & 64.35 & 80.22 & 92.11 & 12.39 & 17.93 & 24.10 & 30.89 & 35.72 \\
Letter & 124.7 & 177.7 & 233.9 & 285.4 & 376.1 & 11.47 & 17.12 & 22.79 & 29.15 & 36.24 \\
shuttle & 212.2 & 292.4 & 382.6 & 487.5 & 610.8 & 33.47 & 47.44 & 65.35 & 80.48 & 105.7 \\
\hline
\end{tabular}

According to the experimental results, for each data set, the computational time of incremental algorithms MIARC, IARC and non-incremental algorithm CAR on different test sets increases with the size of incremental objects. The computational time of incremental algorithms MIARC, IARC is smaller than that of the non-incremental algorithm CAR. Furthermore, this conclusion is best illustrated by the percentage improvement of computational time. In addition, the computational time of incremental algorithm IARC is lower than that of incremental algorithm MIARC. Therefore, the proposed incremental method IARC may be a more reasonable choice for updating the reduction with respect to dynamically incrementing objects.

\subsection{Efficiency analysis}

To verify the effectiveness of the developed incremental algorithms in this subsection. We employ two common evaluation measures in RST to evaluate the decision function of the reductions found by incremental algorithm and non-incremental algorithm. Approximate classified quality (AQ) and approximate classified precision (AP) are defined by Pawlak to depict the precision of approximate classification in RST [25]. If AP and AQ of the feature subset 


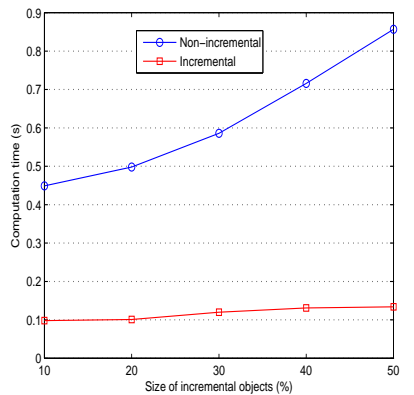

(a) cancer

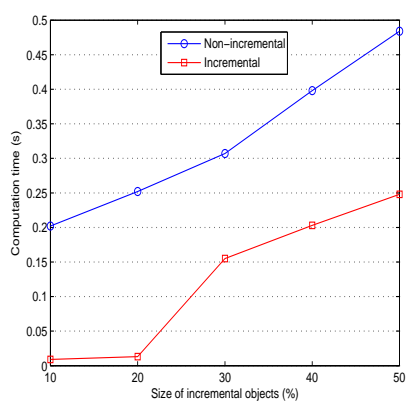

(d) Backup-large

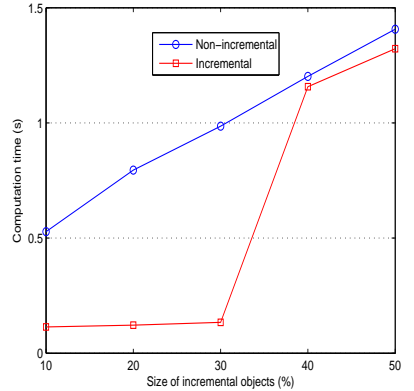

(b) Tic-tea-toe

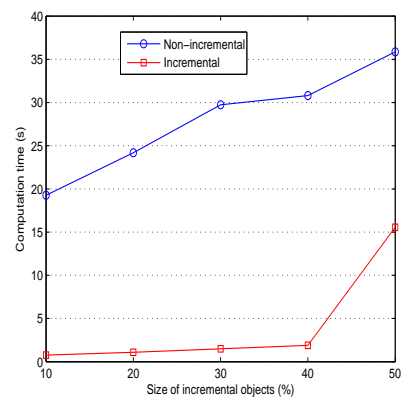

(e) Kr-vs-kp

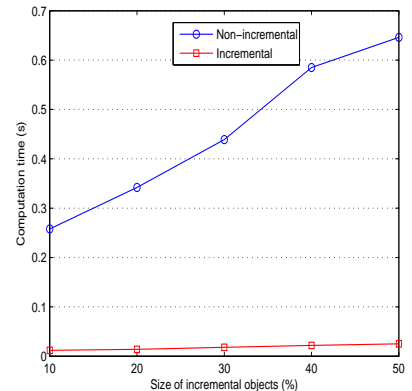

(c) Dermatology

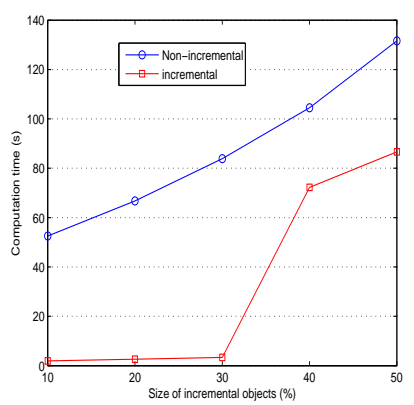

(f) Mushroom

Figure 1: A comparison between non-incremental reduction algorithm CAR and incremental reduction algorithm MIARC.

are identical to those of original attributes, we consider that the feature subset has the same distinguishing power with original decision table. Evaluated results are shown in Table 9.

Definition 17. Given a decision table $S=(U, C \cup D, V, f)$ and $U / D=\left\{X_{1}, X_{2}, \cdots, X_{m}\right\}$. The approximate classified precision of $C$ with respect to $D$ is defined as:

$$
A P_{C}(D)=\frac{\left|\operatorname{POS}_{C}(D)\right|}{\sum_{i=1}^{m}\left|\bar{C} X_{i}\right|}
$$

Definition 18. Given a decision table $S=(U, C \cup D, V, f)$. The approximate classified quality of $C$ with respect to $D$ is defined as:

$$
A Q_{C}(D)=\frac{\left|P O S_{C}(D)\right|}{|U|}
$$

Table 9: A Comparison of Evaluation Measures Based on Knowledge Granularity.

\begin{tabular}{ccccc}
\hline \multirow{2}{*}{ Data sets } & \multicolumn{2}{c}{ CAR } & \multicolumn{2}{c}{ IARC } \\
\cline { 2 - 5 } & AQ & AP & AQ & 1 \\
\hline Cancer & 1 & 1 & 1 & 1 \\
Tic-tea-toe & 1 & 1 & 1 & 1 \\
Kr-vs-kp & 1 & 1 & 1 & 1 \\
Krkopt & 1 & 0.9999 & 1 & 1 \\
Letter & 1 & 1 & 1 \\
shuttle & 1 & & 1 \\
\hline
\end{tabular}

From Table 9, AP and AQ of the feature subset generated by incremental algorithm IARC is very close or identical to that of algorithm CAR on each employed data set. Clearly, reduction generated by incremental algorithm IARC has 


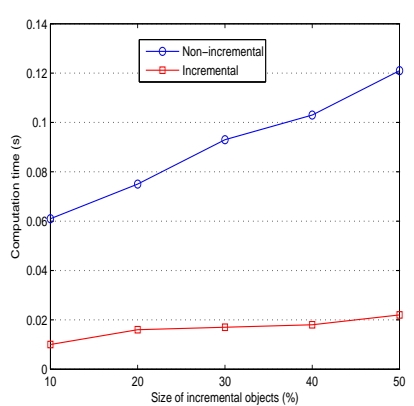

(a) cancer

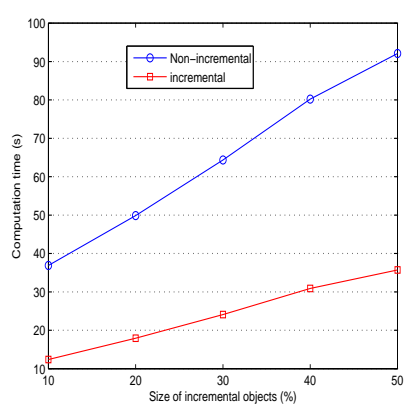

(d) Krkopt

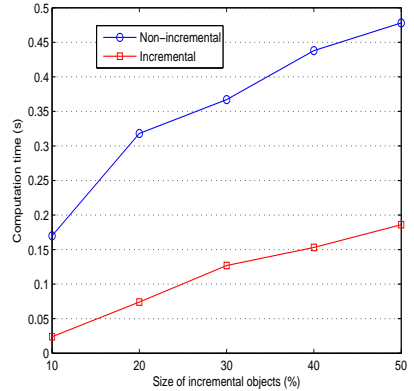

(b) Tic-tea-toe

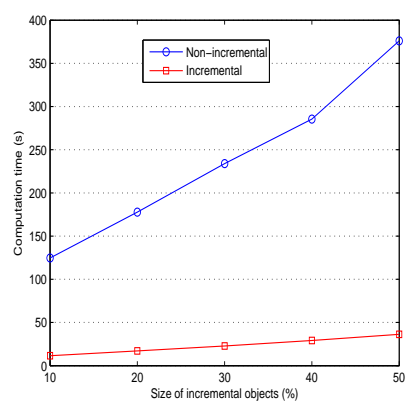

(e) Letter

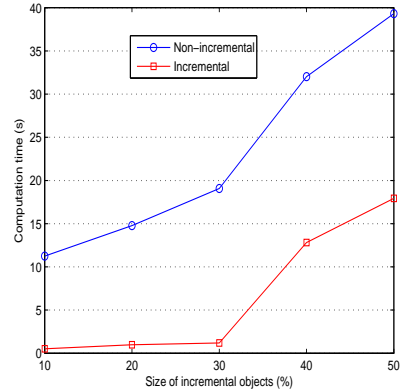

(c) Kr-vs-kp

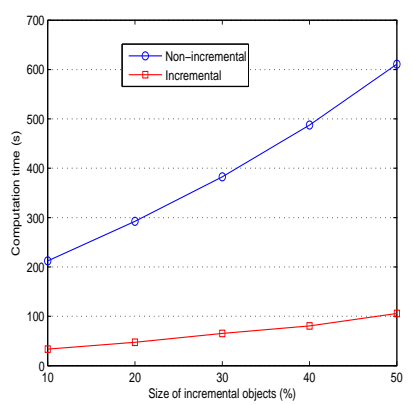

(f) shuttle

Figure 2: A comparison between non-incremental reduction algorithm CAR and incremental reduction algorithm IARC.

almost the same discernible ability with reduction generated by non-incremental algorithm CAR. Hence, the feature subset selected by the proposed incremental reduction method IARC is feasible.

\subsection{Classification accuracy}

We carried on some experiments to compare classification accuracy on reduction algorithms CAR and IARC. The precision of classification is performed on the reductions selected by the proposed reduction methods with 10 -fold cross-validation when $50 \%$ objects of each data set in Table 4 as the basic object set and other $50 \%$ objects as adding object set. In 10-fold cross-validation, the value of average classification accuracy is expressed as a percentage. A given data set is divided into ten parts of equal size. The nine parts are regarded as the training set and a part is regarded as the testing set. Experimental results are listed in Table 10.

Table 10: A Comparison of CAR and IARC on Classification Accuracy.

\begin{tabular}{ccc}
\hline Data sets & CAR & IARC \\
\hline Cancer & 74.7110 & 74.7110 \\
Tic-tea-toe & 69.5198 & 69.5198 \\
Kr-vs-kp & 90.1439 & 91.2052 \\
Krkopt & 35.3258 & 35.3258 \\
Letter & 75.2350 & 76.9412 \\
shuttle & 99.9563 & 99.9563 \\
\hline
\end{tabular}

From Table 10, we can see that the average classification accuracy of the reduction generated by incremental Algorithm IARC is better than that of non-incremental Algorithm CAR in data sets Kr-vs-kp and Letter. The average classification accuracy of incremental Algorithm IARC is identical to that of algorithm CAR on remaining data sets. Hence, the experimental results show that the proposed incremental method IARC is effective on classification tasks. 


\subsection{A comparison with incremental algorithm GIARC}

There are several incremental algorithms for updating the reduction. An incremental reduction algorithm for finding the minimal reduction was presented in [15]. It is only applied to information systems without decision attribute. Two incremental reduction algorithms were developed for decision tables in [29, 30], respectively, however, they are time-consuming. In order to improve the efficiency, Liang et al. proposed a group incremental rough feature selection algorithm based on information entropy [18] and the experimental results showed that it was more efficient than the two algorithms proposed in [29, 30]. Therefore, to further verify the effectiveness and efficiency of incremental reduction algorithm (IARC) based on knowledge granularity, we compare it with the incremental reduction algorithm (GIARC) based on information entropy in [18]. For convenience, classification accuracy is written as CA. The experimental results are shown in Table 11. It is clear that the values of NFS, AQ, AP are very close, and even identical on some data sets. However, CA of IARC is better than that of GIARC and the computational time of IARC is much smaller than that of GIARC except the data set, Kr-vs-kp. Hence, the experimental results show that the incremental algorithm IARC can achieve better performance than incremental algorithm GIARC.

Table 11: A Comparison between Reduction Based on Knowledge Granularity and Reduction Based on Information Entropy.

\begin{tabular}{cccccccccccc}
\hline \multirow{2}{*}{ Data sets } & \multicolumn{3}{c}{ IARC } & \multicolumn{3}{c}{ GIARC } \\
\cline { 2 - 10 } & NFS & Times/s & AQ & AP & CA & NFS & Times/s & AQ & AP & CA \\
\hline cancer & 5 & 0.022 & 1 & 1 & 74.7110 & 4 & 0.057 & 1 & 73.6981 \\
Tic-tea-toe & 8 & 0.186 & 1 & 1 & 69.5198 & 8 & 0.234 & 1 & 0.9999 & 68.6574 \\
Kr-vs-kp & 31 & 17.93 & 1 & 0.9999 & 89.2052 & 29 & 6.522 & 1 & 0.9999 & 88.1101 \\
Krkopt & 6 & 35.72 & 1 & 1 & 35.3258 & 6 & 204.44 & 1 & 0.9999 & 34.4267 \\
Letter & 14 & 36.24 & 1 & 0.9999 & 76.9412 & 12 & 307.8 & 1 & 1 & 72.2457 \\
shuttle & 4 & 105.7 & 1 & 1 & 99.9563 & 4 & 808.6 & 1 & 1 \\
\hline
\end{tabular}

\section{Conclusions}

In practice, many real data in databases may increase dynamically in rows. Incremental reduction algorithms based on knowledge granularity for data sets with dynamically object sets were proposed in the framework of RST. Compared with the non-incremental reduction algorithm based on knowledge granularity and incremental algorithms based on entropies, the proposed incremental algorithms are efficient and effective. This research may shed light on dealing with complicated, large-scale and dynamic data sets in practical applications.

It should be pointed out that updating mechanisms of the knowledge granularity in this paper are applicable when the object set varies. Our future work is to focus on the validation of the proposed approaches in practical applications, study how to extend the proposed algorithms to other extended rough set models and develop approaches to update attribute reduction in information systems with the variation of the attribute set based on knowledge granularity.

\section{Acknowledgements}

This work is supported by NSAF (No. U1230117) and the National Science Foundation of China (Nos. 61175047, 71201133).

\section{References}

[1] V.S. Ananthanarayana, M.M. Narasimha, D. K. Subramanian, Tree structure for efficient data mining using rough sets, Pattern Recognition Letters, 24(6)(2003), pp. 851-862

[2] R.W. Swiniarski, A. Skowron, Rough set methods in feature selection and recognition, Pattern Recognition Letters, 24(6)(2003), pp. 833-849

[3] Y.Y. Yao, Three-way decisions with probabilistic rough sets, Information Sciences, 180(3)(2010), pp. 341-353

[4] Y.Y. Yao, Y. Zhao, Attribute reduction in decision-theoretic rough set models, Information Sciences, 178(17)(2008), pp. 3356-3373

[5] S. Tsumoto, Automated extraction of medical expert system rules from clinical databases based on RST, Information Sciences, 112(14)(1998), pp. 67-84

[6] L. Polkowski, S. Tsumoto, T.Y. Lin (Eds.), Rough set methods and applications, Physica-Verlag, Berlin (2000) 
[7] Y.Y. Yao and N. Zhong, Potential applications of granular computing in knowledge discovery and data mining, Proceedings of the World Multiconference on Systemics, Cybernetics and Informatics, (1999) pp. 573-580

[8] L. Sun, J.C. Xu, Y. Tian, Feature selection using rough entropy-based uncertainty measures in incomplete decision systems, KnowledgeBased Systems, 36(2012), pp. 206-216

[9] C. Chan, A rough set approach to attribute generalization in data mining, Information Sciences, 107(1-4)(1998), pp. 169-176

[10] T. R. Li, D. Ruan, W. Geert, J. Song, Y. Xu, A rough sets based characteristic relation approach for dynamic attribute generalization in data mining, Knowledge-Based Systems, 20(5)(2007), pp. 485-494

[11] J. B. Zhang, T. R. Li, D. Ruan, Z. Z. Gao, C. B. Zhao, Rough sets based matrix approaches with dynamic attribute variation in set-valued information systems, International Journal of Approximate Reasoning, 53(4)(2012), pp. 620-635

[12] Y. Cheng, The incremental method for fast computing the rough fuzzy approximations,Date \& Knowledge Engineering, 70(1)(2011), pp. 84100

[13] F. Wang, J. Y. Liang, Y. H, Qian, Attribute reduction: A dimension incremental strategy, Knowledge-Based Systems, 39(2013), pp. 95-108

[14] S.Y. Li, T.R. Li, D. Liu, Incremental updating approximations in dominance-based rough sets approach under the variation of the attribute set, Knowledge-Based Systems, 40(2013), pp. 17-26

[15] Z. T. Liu, An incremental arithmetic for the smallest reduction of attributes, Acta Electronica Sinica, 27(11)(1999), pp. 96-98 (In Chinese)

[16] M. Orlowska, M. Orlowski, Maintenance of knowledge in dynamic information systems, Slowinski R. (ed.), Intelligent Decision Support. Handbook of Applications and Advances of the RST, Kluwer Academic Publishers, Dordrecht (1992), pp. 315-330

[17] F. Hu, G.Y. Wang, H. Huang, Y. Wu, Incremental attribute reduction based on elementary sets, Proceedings of the 10th International Conference on Rough Sets, Fuzzy Sets, Data Mining and Granular Computing, Regina, Canada (2005), pp. 185-193

[18] J.Y. Liang, F. Wang, C. Y. Dang, Y. H. Qian, A group incremental approach to feature selection applying rough set technique, IEEE Transactions on Knowledge and Data Engineering, 26(2)(2014), pp. 1-31

[19] M. Yang, An incremental updating algorithm of the computation of a core based on the improved discernibility matrix, Chinese Journal of Computers, 29(3)(2006), pp. 407-413 (In Chinese)

[20] H.M. Chen, T.R. Li, D. Ruan, Dynamic maintenance of approximations under a rough-set based variable precision limited tolerance relation, Journal of Multi-valued Logic \& Soft Computing, 18(2012), pp. 577-598

[21] F. Wang, J. Y. Liang, C. Y. Dang, Y. H, Qian, Attribute reduction for dynamic data sets, Applied Soft Computing, 13(1)(2013), pp. 676-689

[22] Z. Y. Xu, Z. P. Liu, B. R. Yang, W. Song, A quickly attribute reduction algorithm with complexity of $\max \left(O(|C||U|), O\left(|C|^{2}|U / C|\right)\right)$, Chinese Journal of Computer, 29(3)(2006), pp. 391-398 (In Chinese)

[23] D. Q. Miao, S. D. Fan, The calculation of knowledge granulation and its application, Systems Engineering-theory \& Practice, 22(1)(2002), pp. 48-56 (In Chinese)

[24] J. C. Xu, J. C. Shi, L. Sun, Attribute reduction algorithm based on relative granularity in decision tables, Computer Science, 36(3)(2002), pp. 205-207 (In Chinese)

[25] Z. Pawlak, Rough sets: Theoretical Aspects of Reasoning about Data, Kluwer Academic Publishers, Boston (1991)

[26] C. Luo, T. R. Li, H. M. Chen, Dynamic Maintenance of Approximations in Set-valued Ordered Decision Systems under the Attribute Generalization, Information Sciences, 257(2014), pp. 210-228

[27] H. M. Chen, T. R. Li, C. Luo, S. J. Horng, G. Y. Wang, A rough set-based method for updating decision rules on attribute values' coarsening and refining, IEEE Transactions on Knowledge and Data Engineering, 26(12)(2014), pp. 2886-2899

[28] W. H. Shu, H. Shen, Updating attribute reduction in incomplete decision systems with the variation of attribute set, International Journal of Approximate Reasoning, 55(3)(2014), pp. 867-884

[29] R. Susmaga, Experiments in incremental computation of reduction, In: Skowron A., Polkowski L. (eds), Rough Sets in Date Mining and Knowledge Discovery, Springer-Verlag, Berlin (1998)

[30] M. Orlowska, M. Orlowski, Maintenance of knowledge in dynamic information systems, In: Slowinski R.(ed.), Intelligent Decision Suppert, Handbook of Applications and Advances of the Rough Set Theory, Kluwer Academic Publishers, Dordrecht (1992), pp. 315-330

[31] L. Wang, T. R. Li, A matrix-based approach for calculation of knowledge granulation, Pattem Recognition and Aitificial Intelligence, 26(5)(2013), pp. 448-453 (In Chinese)

[32] G. Y. Wang, H. Yu. Li, D. C. Yang, Decision table reduction based on conditional information entropy, Chinese Journal of Computer, 25(7)(2002), pp. 759-766 (In Chinese) 